\title{
INTRODUCTION.
}

Under the date of May io, I887, Dr. Arthur Auwers, president of the Astronomische Gesellschaft, extended an invitation to the Naval Observatory to participate in the observation of the stars to the ninth magnitude in the zones $-2^{\circ}$ to $-23^{\circ}$, which undertaking was a continuation of a similar work from $-\mathrm{I}^{\circ}$ to $+80^{\circ}$, then in course of execution under the auspices of this society. In his letter to the Superintendent Dr. A UwERS says, "Since the European observatories from which participation was to be expected lie too far north for a great part of these zones, the cooperation of the better-placed North American observatories is in a high degree desired." In reply to this invitation under date of May 24, I887, the superintendent, Capt. R. L. PhyThian, U. S. N., stated that the Naval Observatory would gladly undertake the observation of a zone of $5^{\circ}$.

Under the date of January I3, I888, a letter was received from Dr. Auwers, announcing the limits of the zone which the Naval Observatory was requested to observe, as follows:

I would request you, therefore, in conformity with the programme in the Vierteljahrsschrift der Astronomischen Gesellschaft, Band IV, Seite $3 \mathrm{O}_{4}$ et seq., and using the zero stars tabulated in the V. J., Band XV, Seite 273 et seq., to have observed with the meridian circle:

'All the stars to the ninth magnitude, inclusive, which, according to the fourth volume of the Bonn Durchmusterung, Bonner Beobachtungen, Band VIII, are found between the parallels $-13^{\circ} 50^{\prime}$ and $-\mathrm{I} 8^{\circ}$. $1 \mathrm{I}^{\prime}$ (for the equinox of $\mathrm{r} 855$ ); and, in addition, all stars of less magnitude than the ninth, which also are contained in the zone observations of LALANDE, BESSEL, ARGELANDER, or LAMONT.'

It will exceed somewhat the programme of the Gesellschaft, but will simplify the preparation of the working list almost as much as it may increase the extent of the observations if, instead of the latter class of stars, you will include in the programme of observation simply:

All stars below the ninth magnitude which are indicated in the Bonn Durchmusterung as having been previously observed at any place.

I should like to recommend this all the more for the Washington zone, as the stars added will mostly be such as are contained in the older Washington zones.

Prof. J. R. Eastman, U. S. N., then in charge of the Pistor \& Martins Transit Circle immediately entered upon the preparations necessary for the execution of the work and in the selection of the stars for observation adopted the suggestion of Dr. AUwERS above quoted of enlarging regular programme. Various circumstances prevented the commencement of the observations until after the removal to the recently completed Naval Observatory on Georgetown Heights, when Assistant Astronomer A. N. Skinner entered upon the prosecution of the work. The working" list prepared by Professor EASTMAN was found to embrace 8,689 stars, thus making an average of 362 for every hour. The actual density of the stars varied throughout 
the hours of right ascension from 7 hours which contained 669 stars to o hours which contained only $25 \mathrm{I}$ stars. To facilitate the work a card catalogue was made, which embodied on a card for each star all the necessary data from the Durchmusterung with blank spaces for the final observed positions, thus permitting easy reference to the details of observation of each star.

The observations are comprised in 236 zones, in all of which the observer at the telescope was Professor Skinner, except in zones 218, 219, and 226 to 23I, inclusive, in which Assistant Astronomer KING performed that duty. The following table exhibits the number of observations comprised in the entire work:

\begin{tabular}{|c|c|c|}
\hline Observer. & Zone stars. & $\begin{array}{c}\text { Zero } \\
\text { and azimuth } \\
\text { stars. }\end{array}$ \\
\hline SkINNER . . . . . & $1958 \mathrm{I}$ & 2050 \\
\hline $\mathrm{K}_{\mathrm{ING}} \ldots \ldots \ldots \ldots$ & 235 & 82 \\
\hline Total....... & $198 \div 6$ & 2132 \\
\hline Grand total. . & & 21948 \\
\hline
\end{tabular}

With a very few exceptions an assistant was present whose duty it was to set the circle, read the two microscopes, and make all the records. Except for six zones the place of assistant was filled either by Frank B. LitTel or Theo I. King. The assistant for zones 227 to $23 \mathrm{I}$, inclusive, was GEORGE K. LAWTON; for zone 226, John C. HAMmond.

Professor Skinner acknowledges his indebtedness to Prof. Frank B. LitTell and Assistant Astronomer THEO I. KING for their valuable assistance in connection with the observations and reductions from the beginning of the work nearly to the time of publication; and also to Prof. W. S. EICHELBERGER, who has supervised the revision of the printer's copy and personally superintended the proof reading.

The first type proof has been read from the original records. All proofs beyond the first have been read only to assure the insertion of the corrections desired, except that in the final plate proof the columns headed Mag., a I9oo.o, $\delta$ Ig0o.o, and $B . D$. have been read entire.

Attention is called to the list of errata for this volume on pages VIII and IX immediately preceding this introduction.

\section{PLAN OF THE WORK.}

The instrument used was the Pistor \& Martins Transit Circle, a complete description of which is found in Publications of the United States Naval Observatory, Second Series, Volume I, pages VII to X. The repairs made in the fall of I899 were carried out in accordance with the statement detailed on page $\mathrm{X}$ above mentioned. The changes and repairs here noted were for the purpose of removing certain defects which existed in the instrument. In addition to the items mentioned in this volume, it was noticed during the latter part of the zone work that determinations of the level constant by the spirit level and nadirs gave evidence of pivot irregularities resulting probably from wear. The pivots were examined in I900, the earliest time 
practicable, with the spherometer caliper, and these measurements confirmed the existence of pivot irregularities of such a character as to render impracticable the application of corrections to the observations. The only remedy is to provide new pivots. Since zero stars have been selected near the parallel of the stars to be determined, it is thought that these instrumental defects will not seriously affect the positions. In reference to the right ascensions, it is much to be regretted that the rate of the observing clock was not at all times as regular as could have been desired.

The plan of the work was made to conform to the published programme of the Astronomische Gesellschaft, V. J. S., Band IV, Seite 304 et seq. In conformity with a suggestion from Dr. Auwers, Professor Skinner decided to read two microscopes in the observation of both zone stars and zero stars. The positions of the zone stars are differentially referred to the catalogue of 303 zero stars selected for the southern zones and published in V. J. S., Band XV, Seite 273 et seq., and the definitive places published in Anhang II Berlin Jahrbuch, Igor.

The scheme of observation involved the following details:

One observation of each zone star in each clamp of the instrument. With few exceptions this was accomplished up to January 4, I896. After this date, as the instrument was principally devoted to other work, it was not deemed advisable to continue this plan.

The reading of two opposite microscopes with each zero star and each zone star.

The observation of each zero star over eleven transit threads and each zone star over five threads.

The making of four bisections with the zenith distance micrometer in case of a zero star and one bisection in case of a zone star.

Usually a group of four zero stars was observed at the beginning of a zone and a similar group at the end. In the case of manyzones zero stars were observed during the progress of the zone.

Except in case of a few of the brightest stars an estimation of the magnitude was made conforming as nearly as possible to the Argelander scale. The observer at the telescope was not furnished with any intimation in reference to the brightness of a star unless the Durchmusterung magnitude showed that it was near the limit of visibility. The result of these estimates seemed to be reasonably self-satisfying for stars of the 8.0 magnitude and fainter. For brighter stars the estimates appear to be very much less accordant. A comparison of the observed magnitudes with those of the Durchmusterung enabled Professor Skinner to detect the variability of four stars during the progress of the zone observations, viz:
B. D. $-14^{\circ} 2893=\mathrm{X}$ Hydrae.
B. D. $-18^{\circ} 3975=$ RT Librae.
B. D. $-16^{\circ} 58 \mathrm{II}=Z$ Capricorni.
B. D. $-15^{\circ} 653 \mathrm{I}=\mathrm{W}$ Ceti.

In the progress of the work several stars were observed not included in the adopted programme. Some of these stars are in the Durchmusterung and some are anonymous. All of these observations have been reduced and are printed in the 
journals of the zones. For convenience of reference the positions of the anonymous stars have been collected in the following table:

Observations of anonymous stars.

\begin{tabular}{|c|c|c|c|c|c|c|c|}
\hline \multirow{2}{*}{$\begin{array}{c}\text { Observed } \\
\text { Mag. }\end{array}$} & \multicolumn{3}{|c|}{$\alpha$ I900. 0} & \multicolumn{3}{|c|}{$\delta$ 1900. o } & \multirow[t]{2}{*}{ Zone. } \\
\hline & h & & $\mathrm{s}$ & & , & & \\
\hline 9. I & I & 47 & $3^{6.92}$ & $-\mathrm{I} 5$ & 26 & 34.02 & I 52 \\
\hline 9.0 & 2 & 54 & $3^{2 .} 64$ & -17 & S & I5. 00 & SI \\
\hline 9.5 & 3 & 57 & 44. 82 & -16 & 20 & 52.48 & I \\
\hline $9 \cdot 3$ & 4 & 55 & 29.69 & $-\mathrm{I} 6$ & 54 & $59.9 \mathrm{I}$ & 159 \\
\hline IO. o & 5 & $5^{6}$ & 34. I 2 & -14 & $3^{\mathrm{I}}$ & 3 I. 36 & I 2 \\
\hline 8.6 & 6 & 15 & 7.25 & -15 & 3 & 55.09 & 14 \\
\hline 9.0 & 7 & 5 & 3I. $9 \mathrm{I}$ & $-\mathrm{I} S$ & 3 & I 4.00 & $9 \mathrm{I}$ \\
\hline$\ldots$ & 7 & I 2 & I3. 49 & -14 & 40 & 42. 44 & 9 \\
\hline 9.5 & 7 & I6 & 45.00 & -16 & 7 & 3 I. 64 & 23 \\
\hline 9. I & 7 & 26 & 37.40 & $-\mathrm{I} 4$ & 45 & $49.4 \mathrm{I}$ & 25 \\
\hline$\ldots$ & 7 & 35 & 0.03 & -16 & 25 & $7 \cdot 4 \mathrm{I}$ & $15 \mathrm{~S}$ \\
\hline 9.8 & s & 28 & บ6. 75 & --16 & 39 & 21.26 & 228 \\
\hline 9.3 & 15 & $3^{2}$ & 7.23 & -15 & 19 & 55.53 & 53 \\
\hline 9.5 & IS & 20 & $47.7 \mathrm{I}$ & -16 & 50 & I3. $\$ 6$ & $6 I$ \\
\hline 9.4 & 20 & 31 & 43.42 & -17 & 12 & I. $2 \mathrm{I}$ & $7 \mathrm{I}$ \\
\hline 9.0 & 20 & & 24. 76 & $-\mathrm{I} 7$ & 50 & 48.00 & 73 \\
\hline
\end{tabular}

THE RIGHT ASCENSIONS.

All the transits were observed chronographically. The arrangement of the transit threads is shown in Table I at the end of this introduction. The method of deriving the instrumental constants is described in Publications of the United States Naval Observatory, Second Series, Volume I. In brief these constants were determined in the following manner:

The azimuth constant.-For zones I to I82 and for zone I84 the azimuth constant was determined by means of the circumpolar stars noted in the reduction tables appended to each zone under the head, Derivation of the Clock Correction and Equator Point. For zone 183 and zones 185 to 236 the azimuth constant was derived by means of the north meridian mark as described in the volume above cited.

The collimation constant. - The collimation constant, which includes the correction for diurnal aberration, was determined at the time of observation by means of the horizontal collimators.

The level constant.-For zones I to 96 and 185 to 236 the level constant was determined by means of the hanging spirit level. For zones 97 to 184 this constant was determined at the nadir by means of the mercury horizon.

Ordinarily one set of constants was determined at the beginning and another at the end of a zone, the mean of which designated as adopted in the reduction tables, was used for both zero stars and zone stars. The constants determined and adopted may be found in the reduction elements for each zone. 
THE DECLINATIONS.

The circle used in these observations is the one designated in the description of the instrument as Circle A. This circle retains the original PisTor \& MARTins divisions. The instrument was set on the nearest even minute and the subdivision of the $2^{\prime}$ interval of the circle graduation was effected by the telescope micrometer. This micrometer is furnished with five zenith threads, two of which are about $8^{\prime \prime}$ apart and are always referred to as the double threads. The line bisecting the space between them is the line of reference for all zenith distance micrometer readings. At a distance of about ten revolutions from the double threads toward the micrometer head is a thread termed $A$, at the same distance on the other side of the double threads is a thread termed $B$, and at about the same distance beyond $B$ is a thread termed C. A ROGERS self-registering apparatus is attached to the head of the zenith distance micrometer screw, enabling the observer to make as many as five bisections which may be recorded at leisure. For a description of this device see Astronomische Nachrichten, Band 63, Seite 77.

The microscopes used in the zone observations were as follows:

\begin{tabular}{|c|c|}
\hline Microscopes. & Zones. \\
\hline VI and VIII & $\left\{\begin{array}{r}I-95 \\
I 66-I 84\end{array}\right.$ \\
\hline II and IV... & $\left\{\begin{array}{r}96-155 \\
157-165\end{array}\right.$ \\
\hline I and III.... & $\left\{\begin{array}{r}156 \\
I 8_{5}-22 I\end{array}\right.$ \\
\hline V and VII... & $222-236$ \\
\hline
\end{tabular}

The adopted value of one revolution of the zenith distance micrometer screw is as follows:

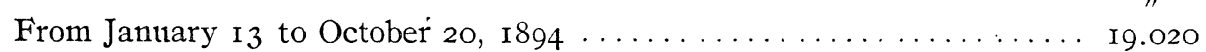

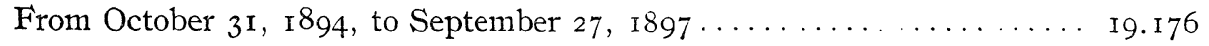

A worn condition of the screw made necessary the adoption of a variable value of the revolution for the period January 27 to July 30 , I900, as is shown in Table IIb.

From October 9, I900, to April 29, I901 ............... I9.1782

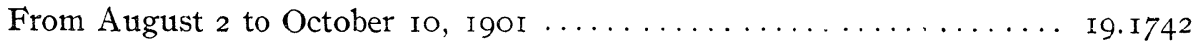

An equator point was deduced from the observation of each zero star and the mean was usually adopted as the equator point of the zone, but in a few zones the equator point was assumed to vary with the time.

The zero stars were selected with the view of having the mean of their declinations fall within the limits of the zone of observation, thus rendering practically insensible the effect of division error and flexure. Consequently no correction for division error or flexure has been introduced. 
At the end of this introduction are the following tables:

TABLE I.-The equatorial thread intervals. The values in these tables are the intervals from the mean of groups b, c, and $d$.

TABLE IIa and IIb.-The adopted values of the readings of the zenith distance micrometer.

TABLE III.-The corrections for inclination and distance of the zenith distance threads. For the zenith distance threads $\mathrm{A}, \mathrm{B}$, and $\mathrm{C}$ the table gives the reduction to the double threads combined with the correction for the inclination of the double threads.

TABLE IV.-The reduction to meridian.

TABLE V.-The precession in declination I894.0 to I900.0.

TABLE VI.-The precession in declination I 895.0 to I900.0.

EXPLANATION OF THE PRINTED PAGES.

The names of the observer and assistant are noted at the head of each zone.

In the journal of each zone:

The column $N o$. gives the number of the zone star in the night's work.

The column Mag. gives the magnitude of the star as estimated by the observer.

The five columns headed Transits give the seconds and tenths of the transit time of each of the five transit threads observed.

The column $G r$. gives the group of transit threads used or the limiting threads in case parts of two groups were used.

The column Mean Thread gives the minutes, seconds and hundredths of the mean of the several transit times reduced to the standard mean thread.

The column Circle Reading gives the observed circle reading for the two microscopes used. The microscopes us " in the zone work are designated in this introduction under the title The Declinaions. When two or more stars were observed at one circle setting, the microscope readings are printed only with the first star of the setting.

The column Telescope Micrometer gives the following particulars:

I. The vertical thread at which the bisection was made.

2. The zenith distance thread used in making the bisection.

3. The complete reading of the zenith distance micrometer.

The column a I90o.o gives the observed right ascension reduced to the epoch I900.0.

The column $\delta$ I900.o gives the observed declination reduced to the epoch I900.0.

In the columns $\alpha 1900.0$ and $\delta$ I900.O brackets indicate results discordant, and therefore not considered in determining the necessary number of observations.

The column $B . D$. gives the Bonn Durchmusterung number for the star observed.

Under the heading Reduction of the Declinations of the Zero Stars appear:

I. The name of the star.

2. The circle reading giving the mean of the two microscopes read.

3. The complete reading for each of the bisections with the zenith distance micrometer.

4. The micrometer equivalent for the mean of the several micrometer readings. 
5. The reduction to meridian.

6. The correction for inclination and zenith distance thread.

7. The correction for refraction.

Under the heading Derivation of the Clock Correction and Equator Point:

The column Name of Star gives the name of the zero stars and azimuth stars.

. The column Mean Thread gives the mean of the times of the several transit threads reduced to the standard mean thread.

The column No. Thds. gives the number of the observed transit threads.

The column Instr. Cor. gives the value of $\mathrm{Aa}+\mathrm{Bb}+\mathrm{Cc}$.

The column Clock Cor. gives the observed clock correction, brackets indicating values not used.

The column Reduced $C$. $R$. gives the sum of the quantities designated 2, 4, 5, 6, and 7 under Reduction of the Declinations of the Zero Stars.

The column Equator Point gives the seconds and tenths of the equator point derived from each zero star. The degrees and minutes may be readily supplied from the adopted equator point. Brackets indicate values not used in forming the adopted equator point.

The following reduction elements are also given:

The adopted clock correction and rate.

The adopted equator point.

The meteorological observations under the following headings:

$B$ indicates barometer.

$t$ indicates attached thermometer.

$T$ indicates external thermometer.

The readings of the barometer and external thermometer have been corrected for errors of scale, which have been determined by careful comparison with the standards of the United States Weather Bureau by courtesy of Prof. C. F. Marvin. The attached thermometer readings have not been corrected for scale error.

In connection with the meteorological data for each zone, in a few cases occurs the designation "Meteorological Journal," which refers to the observatory journal of meteorological readings taken by the watchman every three hours. The instruments are located as follows: The barometer in the hall on the first floor of the main building, and the thermometer in a shelter south of the entrance to the main building.

I6-OI-II 
Under the heading Reduction Tables for the Zone Stars appear the following tables which were prepared with equi-distant intervals of the arguments for zones I to 164 and 185 to 2 I 5 .

\begin{tabular}{|c|c|}
\hline Tables. & Arguments. \\
\hline Instrumental Corrections $=\mathrm{Aa}+\mathrm{Bb}+\mathrm{Cc}$ & Circle Reading. \\
\hline $\begin{array}{l}A=\text { reduction of right ascension to } \\
\text { 1900.0. }\end{array}$ & $\begin{array}{l}\text { FOR zONES I-95. } \\
\text { Apparent right ascension and reduced circle } \\
\text { reading which includes correction for refrac- } \\
\text { tion. }\end{array}$ \\
\hline $\begin{array}{l}D=\text { reduction of zenith distance to the } \\
\text { beginning of the year. }\end{array}$ & $\begin{array}{l}\text { FOR zONES 96-I64 AND I } 85-215 . \\
\text { Apparent right ascension and true zenith dis- } \\
\text { tance which is reduced circle reading with } \\
\text { zenith point correction applied. } \dagger\end{array}$ \\
\hline$R=$ refraction $\ldots \ldots \ldots \ldots \ldots$ & $\left\{\begin{array}{l}\text { Apparent right ascension and reduced circle } \\
\text { reading not corrected for refraction. }\end{array}\right.$ \\
\hline
\end{tabular}

\footnotetext{
*In computing BESSEL's Star-constants, the zenith point correction $-1^{\prime} 30^{\prime \prime}$ has been assumed. The greatest variation from this on any night was $2 \mathrm{I}^{\prime \prime}$.

$\dagger$ In Zones $185^{-215}$, the zenith point correction whose maximum value was $-\mathrm{I}^{\prime} 1 \mathrm{I}^{\prime \prime}$ has been 11 glected. The maximum error resulting is in right ascension $\mathrm{O}^{5} .001$, and in declination $\mathrm{o}^{\prime \prime}$.or.
}

For zones 165 to 184 and 216 to 236 the same quantities are tabulated for each zone star except that $D=$ the reduction to ig0o.0.

Parentheses inclose matter which has been supplied as not existing in the original record or is the result of change from the original record. These omissions or changes are of the following classes:

The designation of the transit threads used.

The vertical thread at which the micrometer bisection was made.

The thread of the zenith distance micrometer used in bisecting.

The degrees and minutes of the circle reading.

It has been the policy not to make changes of a fraction of a revolution of the zenith distance nicrometer reading although an accordant result might follow such changes; for example, changes founded upon the theory of a mistake in the record caused by a similarity of the figures 3 and 5 on the graduated head; an exceptional case, an instance of a change of a half revolution may be found in zone IO2, star II 7 ; but parentheses are used to indicate an interchange of the micrometer readings of two stars or the change of one or more revolutions of the zenith distance screw. 
The following are two examples of the application of the tabular quantities in reduction:

\begin{tabular}{|c|c|c|}
\hline & $\begin{array}{c}\text { Zone } 95 \\
\text { Jaunary 3I, I } 895, \\
\text { Clamp East, } \\
\text { B. D. - I } 3^{\circ} 3476 .\end{array}$ & $\begin{array}{c}\text { Zone } 96, \\
\text { February 26, I895, } \\
\text { Clamp West, } \\
\text { B. D.- I } 4^{\circ} 95^{1} .\end{array}$ \\
\hline & $\mathrm{h} \quad \mathrm{m} \quad \mathrm{s}$ & $\mathrm{h} \quad \mathrm{m} \mathrm{s}$ \\
\hline Mean thread ............ & II $\quad 5$ I 26.75 & $\begin{array}{lll}4 & 39 & 41.82\end{array}$ \\
\hline Instrumental correction . & TI. II & +0.25 \\
\hline Clock correction...$\ldots \ldots$ & -23.86 & $-33.8 \mathrm{I}$ \\
\hline Reduction to $1900.0 \ldots .$. & +I4. II & +53.00 \\
\hline \multirow[t]{2}{*}{$\alpha$ ig00.o. } & II 5 I I8. II & $\begin{array}{lll}4 & 39 & 21.26\end{array}$ \\
\hline & $\circ, 11$ & $\circ, 11$ \\
\hline Circle reading ........ & $\begin{array}{lll}52 & 56 & 9.65\end{array}$ & $\begin{array}{lll}306 & 24 & 9.65\end{array}$ \\
\hline Micrometer equivalent & $+63 \mathrm{I} .3 \mathrm{I}$ & +443.64 \\
\hline Reduction to meridian .... & $+\quad$ r. $6 \mathrm{r}$ & $+\quad 0.02$ \\
\hline Inclination and thread $\ldots$ & $\begin{array}{l}-\quad 2.64 \\
-\quad 10\end{array}$ & $+\quad$ o. I8 \\
\hline Refraction.............. & $+\mathrm{I}$ 22. I5 & - I 2 I. 23 \\
\hline Reduction to $1895.0 \ldots \ldots \ldots$ & $-\quad 4.25$ & $+\quad$ I. 65 \\
\hline Reduction I895.0 to I900.0. . & + I 40.19 & $+\quad 34.60$ \\
\hline $\operatorname{Sum} \ldots \ldots \ldots$ & $\begin{array}{lll}53 & 5 & 3 \mathrm{~S} .02\end{array}$ & $\begin{array}{lll}306 & 28 & 8.5 \mathrm{I}\end{array}$ \\
\hline Equator point .. & $\begin{array}{lll}3^{8} & 56 & 54.78 \\
\end{array}$ & $\begin{array}{lll}.321 & 6 & \text { I } 8.66 \\
\end{array}$ \\
\hline$\delta 1900.0 \ldots$ & $\begin{array}{lll}-14 & \$ & 43.24\end{array}$ & -1438 IO. I5 \\
\hline
\end{tabular}


TABLE I.-The Equatorial Thread Intervals.

\begin{tabular}{|c|c|c|c|c|c|c|c|}
\hline \multirow{2}{*}{\multicolumn{2}{|c|}{$\begin{array}{l}\text { Vertical } \\
\text { Thread. }\end{array}$}} & Jan. I3, IS94, tc & Oct. $3 \mathrm{I}, \mathrm{I} 894$, to & Feb. 26, 1895, t & o June 29, I 896 , to & Jan. 27,1900 , to & Nov. 2I, 1900, to \\
\hline & & Oct. 20 , I894. & Jan. 31, I895. & & Sept. 27,1897 . & Nov. I 2, 1900. & \\
\hline \multirow{2}{*}{\multicolumn{2}{|c|}{ I }} & $\mathrm{s}$ & $\mathrm{s}$ & $\mathrm{s}$ & $\mathrm{s}$ & $\mathrm{s}$ & $\mathbf{s}$ \\
\hline & & +36.709 & +37.010 & $+37.08 \mathrm{r}$ & +37.010 & +37.090 & +37.046 \\
\hline & $a_{x}$ & 32.070 & 32.306 & 32.469 & 32.306 & 32. 495 & $32.33 I$ \\
\hline & $a_{2}$ & 30.349 & 30.568 & 30.544 & 30.568 & 30.543 & 30.576 \\
\hline & $a_{3}$ & 28.490 & 28.683 & 28. 756 & 28.683 & 28. 754 & 28.706 \\
\hline & $a_{4}$ & 26.600 & 26. 802 & 26. 970 & 26.802 & 26. 969 & 26. 797 \\
\hline II & $a_{5}$ & $24.5 \mathrm{IO}$ & 24.678 & 24. 734 & 24.678 & 24. 7I4 & 24.682 \\
\hline \multirow[t]{5}{*}{ III } & $b_{1}$ & I2. 223 & I2. 334 & 12. 388 & I2. 334 & I2. 389 & I2. 356 \\
\hline & $b_{2}$ & 9. 694 & 9. 782 & 9. $7 \mathrm{I} 8$ & 9. 782 & 9.727 & 9.775 \\
\hline & $b_{3}$ & 8. 164 & 8. 227 & 8. 236 & 8. 227 & 8.227 & 8. 237 \\
\hline & $c_{x}$ & 4. II 8 & 4. 136 & 4. 154 & 4. 136 & 4. I63 & 4. I 43 \\
\hline & $c_{2}$ & 2. II 4 & +2.112 & 2. 078 & +2.112 & 2. 090 & +2.119 \\
\hline \multirow[t]{5}{*}{ IV } & $c_{3}$ & +0.002 & -0.017 & +0.017 & -0.017 & +0.032 & -0.032 \\
\hline & $\mathrm{c}_{4}$ & -2.054 & 2. 078 & - 2. II 2 & 2. 078 & - 2. II9 & 2. 090 \\
\hline & $c_{5}$ & 4. 120 & 4. I.54 & 4. 136 & 4. I 54 & 4. I43 & 4. $I_{6}$ \\
\hline & $d_{x}$ & 8. 186 & 8. 236 & 8.227 & 8.236 & 8.237 & 8.227 \\
\hline & $d_{2}$ & 9.664 & 9. 718 & 9. 782 & 9.718 & 9.775 & 9.727 \\
\hline $\mathrm{V}$ & $d_{3}$ & I2. 29I & 12. 388 & I2. 334 & I2. 388 & I2. 356 & I2. $3^{89}$ \\
\hline \multirow[t]{5}{*}{ VI } & $e_{x}$ & 24.550 & 24. 734 & 24.678 & 24. 734 & 24.682 & 24. 7I4 \\
\hline & $\mathrm{e}_{2}$ & 26. 755 & 26.970 & $26.8 \mathrm{o} 2$ & 26. 970 & 26. 797 & 26.969 \\
\hline & $e_{3}$ & $28.53 \mathrm{I}$ & 28. $75^{6}$ & 28.683 & 28. 756 & 29. 706 & 28. 754 \\
\hline & $\mathrm{e}_{4}$ & 30.320 & 30.544 & 30. 568 & 30. 544 & 3ว. 576 & 30.543 . \\
\hline & $e_{5}$ & 32.223 & 32.469 & 32.306 & 32.469 & $3^{2} .33^{I}$ & 32.495 \\
\hline VII & & -36.802 & $-37.08 \mathrm{I}$ & -37.010 & $-37.08 \mathrm{r}$ & -37.046 & -37.090 \\
\hline
\end{tabular}


TABLE IIa.-The Adopted Values of the Readings of the Zenith Distance Micrometer.

\begin{tabular}{|c|c|c|c|c|c|c|c|c|c|c|c|c|}
\hline & \multicolumn{3}{|c|}{$\begin{array}{c}\text { Jan. I3, I894, to Oct. 20, } \\
\text { I894. }\end{array}$} & \multicolumn{3}{|c|}{$\begin{array}{c}\text { Oct. } 31, \text { I894, to Sept. } 27, \\
\text { 1897. }\end{array}$} & \multicolumn{3}{|c|}{$\begin{array}{c}\text { Oct. 9, I900, to Apr. 29, } \\
\text { I90I. }\end{array}$} & \multicolumn{3}{|c|}{$\begin{array}{l}\text { Aug. 2, Igor, to Oct. Io, } \\
\text { Igor. }\end{array}$} \\
\hline & $4^{\prime}$ & $5^{\prime}$ & $6^{\prime}$ & $4^{\prime}$ & $5^{\prime}$ & $6^{\prime}$ & $3^{\prime}$ & $4^{\prime}$ & $5^{\prime}$ & $3^{\prime}$ & $4^{\prime}$ & $5^{\prime}$ \\
\hline 'I & revs. & revs. & revs. & revs. & revs. & revs. & revs. & revs. & revs. & revs. & revs. & revs. \\
\hline o & 40.000 & 43. I55 & 46.309 & 40.000 & 43. 129 & $46.25^{8}$ & $39.3^{86}$ & 42. 514 & 45.643 & 39.388 & 42. 5I7 & 45.646 \\
\hline I & .053 & .207 & .362 & .052 & $.18 \mathrm{I}$ & .310 & .438 & .566 & .695 & .440 & .569 & .698 \\
\hline 2 & .105 & .260 & $.4 \mathrm{I} 4$ & .104 & .233 & .362 & .490 & 618 & .747 & .492 & $.62 \mathrm{I}$ & .750 \\
\hline 3 & .158 & .312 & .467 & .156 & .285 & .414 & .542 & $.67 \mathrm{I}$ & .799 & .544 & .673 & .802 \\
\hline 4 & .210 & .365 & .519 & .209 & .337 & .466 & .594 & .723 & $.85 \mathrm{I}$ & .596 & .725 & .855 \\
\hline 5 & 40.263 & $43.4 \mathrm{I} 7$ & 46.572 & $40.26 \mathrm{I}$ & 43.390 & 46.518 & 39.646 & 42. 775 & 45.903 & 39.648 & 42.778 & 45.907 \\
\hline 6 & .315 & .470 & .625 & .313 & .442 & .571 & .699 & .827 & 45.956 & $.7 \mathrm{OI}$ & .830 & 45.959 \\
\hline 7 & $\cdot 368$ & .523 & .677 & .365 & .494 & .623 & $.75 \mathrm{I}$ & .879 & 46. 008 & .753 & .882 & 46. OII \\
\hline 8 & $.42 \mathrm{I}$ & .575 & .730 & .417 & .546 & .675 & .803 & $.93 \mathrm{I}$ & .060 & .805 & .934 & .063 \\
\hline 9 & .473 & .628 & .782 & .469 & .598 & .727 & .855 & 42. 983 & . II 2 & .857 & 42. 986 & . II5 \\
\hline IO & 40.526 & 43.680 & 46.835 & $40.52 \mathrm{I}$ & 43.650 & 46. 779 & 39.907 & 43.036 & 46. I64 & 39.909 & 43.038 & 46. I68 \\
\hline II & .578 & .733 & .887 & .574 & .702 & .831 & 39. 959 & .088 & .216 & $39.96 \mathrm{I}$ & .091 & .220 \\
\hline I 2 & $.63 \mathrm{I}$ & .785 & .940 & .626 & .755 & $.88_{3}$ & 40. OI I & .140 & .268 & 40.013 & .143 & .272 \\
\hline I3 & .683 & .838 & 46. 993 & .678 & .807 & .936 & .064 & .192 & .321 & .066 & .195 & .324 \\
\hline I4 & .736 & $.89 \mathrm{I}$ & 47.045 & .730 & .859 & 46. 998 & .116 & .244 & .373 & . II 8 & .247 & .376 \\
\hline 15 & 40. 789 & 43.943 & 47.098 & 40.782 & $43.9 \mathrm{II}$ & 47.040 & 40. 168 & 43. 296 & 46. 425 & 40. 170 & 43. 299 & 46. 428 \\
\hline 16 & $.84 \mathrm{I}$ & 43.996 & .150 & .834 & 43.963 & .092 & .220 & .348 & .477 & .222 & $.35 \mathrm{I}$ & .480 \\
\hline I 7 & .894 & 44.048 & .203 & .887 & 44 . or 5 & . I44 & .272 & $.40 \mathrm{I}$ & .529 & .274 & .403 & .533 \\
\hline 18 & .946 & . IOI & .256 & .939 & .068 & .196 & .324 & .453 & $.5^{85}$ & .326 & .456 & .585 \\
\hline I9 & 40. 999 & .154 & .308 & 40. $99 \mathrm{I}$ & . I2O & .249 & .376 & .505 & .633 & .379 & .508 & .637 \\
\hline 20 & 41. 052 & 44.206 & $47.36 \mathrm{I}$ & 4r. 043 & 44. I 72 & $47.3 \mathrm{OI}$ & 40.428 & 43.557 & 46.686 & $40.43 \mathrm{I}$ & 43.560 & 46.689 \\
\hline 2I & .104 & .259 & $.4 \times 3$ & .095 & .224 & .353 & $.48 \mathrm{I}$ & .609 & .738 & .483 & .612 & $.74 \mathrm{I}$ \\
\hline 22 & . 557 & .311 & .466 & .147 & .276 & .405 & .533 & $.66 \mathrm{I}$ & .790 & .535 & .664 & .793 \\
\hline 23 & .209 & .364 & $.5^{18} 8$ & . 199 & .328 & .457 & .585 & .713 & .842 & .587 & .716 & .846 \\
\hline 24 & .262 & .416 & $.57 \mathrm{I}$ & .252 & .380 & .509 & .637 & .766 & .894 & .639 & .768 & .898 \\
\hline 25 & 4I. 3 I 4 & 44.469 & 47.624 & 4I. 304 & 44.433 & $47.56 \mathrm{I}$ & 40.689 & 43. $8 \mathrm{I} 8$ & 46.946 & $40.69 \mathrm{I}$ & 43.821 & 46. 950 \\
\hline 26 & .367 & .522 & .676 & $.35^{6}$ & .485 & .614 & $.74 \mathrm{I}$ & .870 & $46.99^{8}$ & .744 & .873 & 47.002 \\
\hline 27 & .420 & .574 & .729 & .408 & .537 & .666 & .793 & .922 & $47.05 \mathrm{I}$ & .796 & .925 & .054 \\
\hline 28 & .472 & .627 & $.78 I$ & .460 & .589 & .718 & .846 & 43.974 & . 103 & .848 & 43.977 & . 106 \\
\hline 29 & .525 & .679 & .834 & .512 & $.64 \mathrm{r}$ & .770 & .898 & 44.026 & .155 & .900 & 44. 029 & .158 \\
\hline 30 & 4I. 577 & 44. 732 & 47.886 & 4I. 564 & 44.693 & 47.822 & 40.950 & 44.078 & 47.207 & 40. 952 & 44. $08 \mathrm{I}$ & $47.2 \mathrm{II}$ \\
\hline $3 \mathrm{I}$ & .630 & .784 & .939 & .617 & .745 & .874 & 4I. 002 & $.13 \mathrm{I}$ & .259 & 4I. 004 & . I34 & .263 \\
\hline 32 & .682 & .837 & 47. 992 & .669 & .798 & .926 & .054 & .183 & $.3 \mathrm{II}$ & .057 & .186 & .315 \\
\hline 33 & .735 & . 890 & 48.044 & $.72 \mathrm{I}$ & .850 & 47.979 & . 10 & .235 & $.3^{63}$ & .109 & .238 & .367 \\
\hline 34 & .788 & .942 & .097 & .773 & .902 & 48. O3I & .158 & .287 & .416 & $\therefore 6 \mathrm{I}$ & .290 & .419 \\
\hline 35 & 4I. 840 & 44. 995 & 48. I 49 & 4I. 825 & 44. 954 & 48.083 & 4I. 2II & 44.339 & 47.468 & 4I. $2 \mathrm{I} 3$ & $44 \cdot 342$ & $47.47 \mathrm{I}$ \\
\hline 36 & 893 & 45.047 & .202 & .877 & 45.006 & .135 & .263 & $.39 \mathrm{I}$ & $.5^{20}$ & .265 & $\cdot 394$ & .524 \\
\hline 37 & .945 & . 100 & .254 & .929 & .058 & . I87 & .315 & .443 & .572 & $\cdot 317$ & .446 & .576 \\
\hline 38 & 4I. 998 & .152 & .307 & 4I. 982 & . IIO & .239 & .367 & .496 & .624 & .369 & .499 & .628 \\
\hline 39 & 42.050 & .205 & .360 & 42. 034 & .163 & $.29 \mathrm{I}$ & .419 & .548 & .676 & .422 & .551 & .680 \\
\hline 40 & 42. $\mathrm{IO} 3$ & $45.25^{8}$ & 48. 4I 2 & 42.086 & $45.2 \mathrm{I} 5$ & 48. 344 & 4I. $47 \mathrm{I}$ & 44.600 & 47.728 & 4I. 474 & 44.603 & 47.732 \\
\hline $4 \mathrm{I}$ & .156 & . 310 & .465 & .138 & .267 & .396 & $.5^{23}$ & .652 & $.78 \mathrm{r}$ & $.5^{26}$ & .655 & .784 \\
\hline 42 & .208 & .363 & .517 & . I9o & .319 & .448 & .576 & .704 & .833 & .578 & .707 & .836 \\
\hline 43 & $.26 \mathrm{I}$ & .415 & .570 & .242 & $.37 \mathrm{I}$ & .500 & .628 & .756 & .885 & .630 & .759 & .889 \\
\hline 44 & .313 & .468 & .623 & .294 & .423 & .552 & .680 & .808 & .937 & .682 & .812 & $.94 \mathrm{I}$ \\
\hline 45 & 42.366 & $45.52 \mathrm{I}$ & 48.675 & 42. 347 & 45.475 & 48.604 & 4I. 732 & $44.86 \mathrm{I}$ & 47. 989 & 4I. 735 & 44.864 & 47.993 \\
\hline 46 & .419 & .573 & .728 & .399 & .528 & .656 & .784 & .913 & 48. $04 \mathrm{I}$ & .787 & .916 & 48. 045 \\
\hline 47 & .471 & .626 & .780 & .4 & $.5^{80}$ & .709 & .836 & 44. 965 & .093 & .839 & 44. 968 & .097 \\
\hline 48 & .524 & .678 & .833 & .503 & .632 & $.76 \mathrm{I}$ & .888 & 45.017 & .146 & $.89 \mathrm{I}$ & 45.020 & .149 \\
\hline 49 & .576 & $.73 I$ & .885 & .555 & .684 & .813 & $.94 \mathrm{I}$ & .069 & . I98 & .943 & .072 & .202 \\
\hline 50 & 42.629 & $45 \cdot 783$ & 48. 938 & 42. 607 & 45.736 & 48.865 & 4I. 993 & 4.5. $12 \mathrm{I}$ & 48.250 & 4I. 995 & 45. 124 & 48.254 \\
\hline $5^{\mathrm{I}}$ & $.68 \mathrm{I}$ & .836 & 48. $99 \mathrm{I}$ & .660 & .788 & .917 & 42.045 & . I 73 & .302 & 42. 0.47 & .177 & .306 \\
\hline $5^{2}$ & .734 & .889 & 49.043 & .712 & $.84 \mathrm{I}$ & 48.969 & .097 & .226 & .354 & . 100 & .229 & $.35^{8}$ \\
\hline 53 & .787 & $.94 I$ & .096 & .764 & .893 & 49. 022 & .149 & .278 & .406 & . 152 & $.28 \mathrm{I}$ & . $4 \mathrm{IO}$ \\
\hline 54 & .839 & 45. 994 &.$T 48$ & .816 & .945 & .074 & $.20 \mathrm{I}$ & .330 & $.45^{8}$ & .204 & $\cdot 333$ & .462 \\
\hline 55 & 42.892 & 46. 046 & 49. $2 \mathrm{OI}$ & 42. 868 & 45. 997 & 49. 126 & 42. 253 & $45 \cdot 3^{82}$ & $48.5^{\mathrm{II}}$ & 42. 256 & $45 \cdot 3^{85}$ & $48.5 \mathrm{I} 4$ \\
\hline 56 & .944 & .099 & .253 & .920 & 46. 049 & .178 & .306 & .434 & .563 & $\cdot 308$ & .437 & .567 \\
\hline 57 & 42. 997 & . I5I & .306 & 42.972 & . IOI & .230 & $.35^{8}$ & .486 & .615 & .360 & .490 & .619 \\
\hline $5^{8}$ & 43. 049 & . 204 & .359 & 43.025 & . I53 & .282 & .410 & .538 & .667 & $.4 \mathrm{I} 3$ & .542 & $.67 \mathrm{I}$ \\
\hline 59 & .102 & .257 & $.4 \mathrm{II}$ & .077 & .206 & .334 & .462 & $.59 \mathrm{I}$ & .719 & .465 & .594 & .723 \\
\hline 60 & 43. I55 & 46.309 & 49.464 & 43. 129 & 46. $25^{8}$ & 49. 387 & 42. $5^{14}$ & 45.643 & 48. $77 \mathrm{I}$ & 42.517 & 45.646 & 48. 775 \\
\hline
\end{tabular}


TABLE IIb.-The Adopted Values of the Readings of the Zenith Distance Micrometer.

\begin{tabular}{|c|c|c|c|c|c|c|c|c|c|c|}
\hline \multirow{2}{*}{ Revs. } & \multicolumn{10}{|c|}{ Jan. 27 , I900, to July 30, I900. } \\
\hline & .0 &.$I$ & .2 & .3 & .4 & $\cdot 5$ & . .6 & .7 & .8 & $\cdot 9$ \\
\hline & , " & " & , "I & , "1 & , II & , II & , II & , il & , "I & \\
\hline 30 & o o. @ & O I. 92 & o $\quad 3 \cdot \Omega_{3}$ & o 5.75 & o 7.67 & o $9.5^{8}$ & O II. 50 & $\mathrm{O} I 3 \cdot 4 \mathrm{I}$ & $\mathrm{O} 15.33$ & o $\mathrm{I} 7.25$ \\
\hline $3 I$ & I9. I6 & $2 \mathrm{I} . \mathrm{OS}$ & 22.99 & 24. $9 \mathrm{I}$ & 26. 82 & 28. 73 & 30.65 & 32.56 & 34.48 & 36.39 \\
\hline $3^{2}$ & $3^{S .} 3^{I}$ & 40.22 & 42. I3 & 44.05 & $45 \cdot 96$ & 47.87 & 49. 78 & $5 \mathrm{I} \cdot 7 \mathrm{O}$ & $53.6 \mathrm{I}$ & $55 \cdot 5^{2}$ \\
\hline 33 & $57 \cdot 43$ & $59 \cdot 34$ & I I. 25 & I 3.16 & I 5.07 & I 6.98 & I 8.89 & I Io. 80 & I $12.7 \mathrm{I}$ & I I 4.62 \\
\hline 34 . & I 16.53 & I IS. 44 & 20.35 & 22. 26 & 24. I7 & 26. o8 & 27.99 & 29. 89. & 31.80 & 33.71 \\
\hline 35 & 35.62 & $37 \cdot 5^{2}$ & 39.43 & $41 \cdot 34$ & 43.24 & 45. I5 & 47.06 & 48. 96 & 50.87 & 52. 78 \\
\hline 36 & 54.68 & 56.59 & 58.49 & $2 \quad 0.40$ & $2 \quad 2.30$ & 24.20 & $26 . \mathrm{II}$ & 28.01 & 29.92 & 2 I I. 82 \\
\hline 37 & $213 \cdot 72$ & $2 \mathrm{I} 5.62$ & $2 I 7.53$ & I9. 43 & 2 I. 33 & 23.23 & 25.13 & 27.04 & 28. 94 & 30.84 \\
\hline $3^{8}$ & 32. 74 & 34.64 & 36.54 & 38.44 & 40.34 & 42. 24 & 44. I4 & 46.04 & 47.94 & 49.84 \\
\hline 39 & 51. 73 & 53.63 & 55.53 & 57.43 & 59.33 & 3 x. 23 & 33.12 & 35.02 & 36.92 & 38.82 \\
\hline 40 & 3 10. 72 & $3 \mathrm{r} 2.6 \mathrm{I}$ & $3 \mathrm{I} 4.5 \mathrm{I}$ & $3 \mathrm{I} 6.4 \mathrm{I}$ & $3 \mathrm{I} 8.3 \mathrm{I}$ & 20. 20 & 22. 10 & 24. 00 & 25.90 & 27.80 \\
\hline $4 \mathrm{I}$ & 29. 70 & 3I. 59 & 33.49 & 35.39 & 37.29 & 39. 19 & 4I. 09 & 42. 99 & 44.89 & 46. 79 \\
\hline 42 & 48. 70 & 50.60 & 52. 50 & 54.40 & $56.3 \mathrm{I}$ & $5^{\text {S. } 2 \mathrm{I}}$ & 4 o. I2 & $4 \quad 2.02$ & $4 \quad 3.93$ & $\begin{array}{ll}4 & 5.83\end{array}$ \\
\hline 43 & $4 \quad 7.74$ & 49.64 & 4 II. 55 & 4 I 3.46 & $4 \quad 15.37$ & $4 \quad 17.28$ & I9. I9 & 2I. IO & 23. OI & 24. 92 \\
\hline 44 & 26. 84 & 2S. 75 & 30.66 & $3^{2} \cdot 5^{8}$ & 34.49 & $36.4 \mathrm{I}$ & 38.33 & 40. 24 & 42. I6 & 44. 08 \\
\hline 45 & 46. 00 & $47 \cdot 9 \mathrm{I}$ & $49.8_{3}$ & 5I. 75 & 53.67 & 55.59 & $57 \cdot 5^{2}$ & 59.44 & 5 I. 36 & $\begin{array}{ll}5 & 3.28\end{array}$ \\
\hline 46 & $5 \quad 5.20$ & $5 \quad 7 \cdot \mathrm{I}_{3}$ & $5 \quad 9.05$ & 5 ro. 98 & 5 I 2.90 & $5 \mathrm{I} 4.82$ & 516.75 & 5 I8. 68 & 20. 60 & 22.53 \\
\hline 47 & 24.45 & $26.3^{8}$ & 28.31 & 30.24 & 32. 16 & 34. 09 & 36.02 & 37.95 & 39.88 & 4I. 8I \\
\hline 48 & 43. 74 & 45.67 & 47.60 & 49. 53 & 5I. 47 & 53.40 & $55 \cdot 33$ & 57.26 & 59. I9 & 6 I. I3 \\
\hline 49 & 63.06 & $6 \quad 4.99$ & $\begin{array}{ll}6 & 6.93\end{array}$ & 6 S.86 & 6 10. 79 & 6 I2. 73 & 6 I 4.66 & 616.60 & $6 \times 8.53$ & 20.47 \\
\hline 50 & 22. 40 & 24. 34 & 26.27 & 28. 20 & 30. 14 & 32.07 & 34 or & $35 \cdot 94$ & 37.87 & $39.8 \mathrm{r}$ \\
\hline $5^{\mathrm{I}}$ & 4I. 74 & 43.67 & 45.60 & $47 \cdot 54$ & 49. 47 & 5I. $4 \mathrm{O}$ & $53 \cdot 33$ & 55.26 & 57.20 & 59. I3 \\
\hline $5^{2}$ & 7 I. 06 & $\begin{array}{ll}7 & 2.99\end{array}$ & $7 \quad 4.92$ & $7 \quad 6.85$ & $7 \quad 8.78$ & 7 Iо. $7 \mathrm{I}$ & $7 \quad 12.64$ & 7 I 4.57 & 716.50 & $7 \quad 18.42$ \\
\hline 53 & 20.35 & 22. 28 & 24. 2 I & 26. I 4 & 28. 07 & 29. 99 & 3I. 92 & 33.85 & $35 \cdot 77$ & 37.70 \\
\hline 54 & 39.63 & 4I. 55 & 43.48 & 45.40 & 47.33 & 49. 26 & 5I. I8 & 53. 1о & 55.03 & 56.95 \\
\hline 55 & 58.88 & 8 o. 80 & $8 \quad 2.73$ & $8 \quad 4.65$ & $\begin{array}{ll}8 & 6.57\end{array}$ & $8 \quad 8.50$ & 8 I0. 42 & 812.34 & 8 I4. 26 & 8 I6. I8 \\
\hline 56 & 8 I8. II & 20. 03 & 2I. 95 & 23. 87 & 25. 79 & $27.7 \mathrm{I}$ & 29.63 & 3I. 55 & 33.47 & 35.39 \\
\hline 57 & $37 \cdot 3 \mathrm{I}$ & 39.23 & 4I. I 5 & 43.07 & 44.99 & 46.90 & 48.82 & 5०. 74 & 52.66 & $54.5^{8}$ \\
\hline 58 & 56.50 & $5^{8.42}$ & $9 \quad 0.33$ & $9 \begin{array}{ll}9.25\end{array}$ & 94.17 & 96.09 & 98.00 & $9 \quad 9.92$ & 9 Ir. 83 & $9 \mathrm{I} 3.75$ \\
\hline 59 & 915.66 & $917.5^{8}$ & I9. 49 & 2I. $4 \mathrm{I}$ & $23 \cdot 32$ & 25.24 & 27. I 5 & 29. 06 & 30. 98 & 32.89 \\
\hline
\end{tabular}


TABLE III.-Inclination and Distance of Zenith Distance Threads.

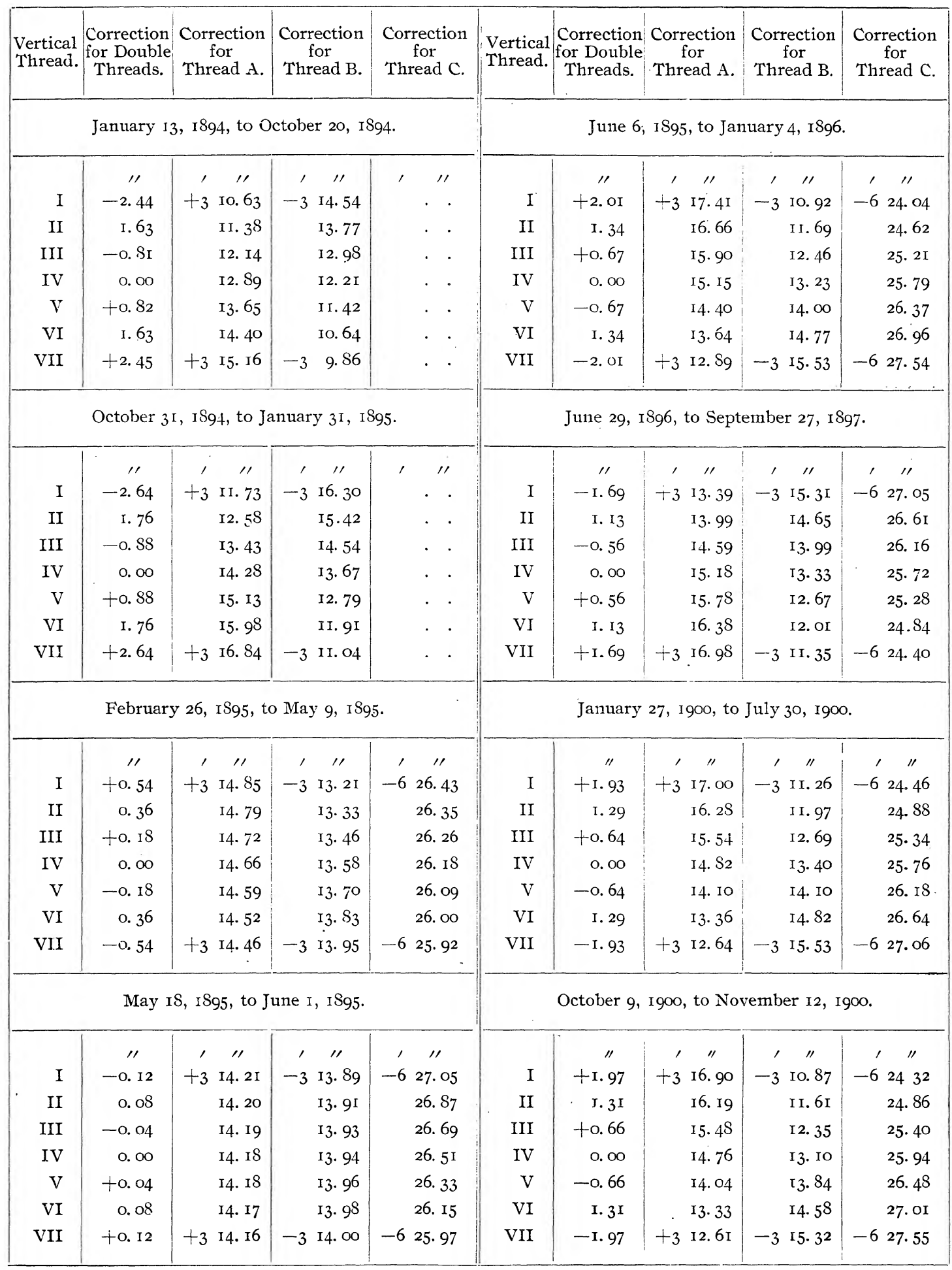


TABLE III.-Inclination and Distance of Zenith Distance Threads-Continued.

\begin{tabular}{|c|c|c|c|c|c|c|c|c|c|}
\hline $\begin{array}{l}\text { Vertical } \\
\text { Thread. }\end{array}$ & $\begin{array}{l}\text { Correction } \\
\text { for Double } \\
\text { Threads. }\end{array}$ & $\begin{array}{l}\text { Correction } \\
\text { for } \\
\text { Thread A. }\end{array}$ & $\begin{array}{l}\text { Correction } \\
\text { for } \\
\text { Thread B. }\end{array}$ & $\begin{array}{l}\text { Correction } \\
\text { for } \\
\text { Thread C. }\end{array}$ & $\begin{array}{l}\text { Vertical } \\
\text { Thread. }\end{array}$ & $\begin{array}{c}\text { Correction } \\
\text { for Double } \\
\text { Threads. }\end{array}$ & $\begin{array}{l}\text { Correction } \\
\quad \text { for } \\
\text { Thread A. }\end{array}$ & $\begin{array}{l}\begin{array}{c}\text { Correction } \\
\text { for } \\
\text { Thread B. }\end{array}\end{array}$ & $\begin{array}{l}\text { Correction } \\
\text { for } \\
\text { Thread C. }\end{array}$ \\
\hline \multicolumn{5}{|c|}{ November 2I, I900, to December I5, I900. } & \multicolumn{5}{|c|}{ December 20 , I900, to October Io, I90I. } \\
\hline I & $\begin{array}{c}\prime \prime \\
-2.09\end{array}$ & $\begin{array}{c}1 " 11 \\
+3 \text { I } 2.49\end{array}$ & $\begin{array}{cc}\prime \prime \prime \\
-3 & 15.44\end{array}$ & 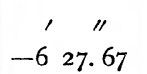 & I & $\begin{array}{c}\prime \prime \\
-2.26\end{array}$ & $\begin{array}{c}\prime \prime \prime \\
+312.38\end{array}$ & $\begin{array}{cc}\prime \prime \prime \\
-3 & 15.67\end{array}$ & 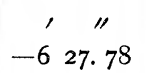 \\
\hline II & I. 39 & I3. 25 & I 4.65 & 27.09 & II & I. 50 & I3. 18 & I 4.87 & 27. 16 \\
\hline III & -0.70 & I 4.00 & I3. 88 & $26.5^{2}$ & III & -0.75 & I3. 97 & I4. 07 & 26.55 \\
\hline IV & 0.00 & I4. 76 & I3. Io & 25.94 & IV & o. oo & $14 \cdot 76$ & 13.27 & 25.94 \\
\hline $\mathrm{V}$ & +0.70 & I $5.5 \mathrm{I}$ & I2. 32 & $25 \cdot 3^{6}$ & $\mathrm{~V}$ & +o. 75 & $\mathrm{I} 5 \cdot 55$ & I2. 47 & $25 \cdot 33$ \\
\hline VI & I. 39 & I6. 26 & II. 54 & 24. 78 & VI & I. 50 & I6. 35 & II. 67 & 24. 72 \\
\hline VII & +2.09 & +317.02 & -3 ro. 75 & $-624 \cdot 20$ & VII & +2.26 & +3 I7. 15 & -3 го. 86 & -624 . IO \\
\hline
\end{tabular}

TABLE IV.-Reduction to Meridian.

\begin{tabular}{|c|c|c|c|c|c|c|c|c|c|c|c|c|c|c|c|}
\hline \multicolumn{4}{|c|}{$\begin{array}{l}\text { Jan. I3, r894, to Jan. } 3 \mathrm{r}, \\
\text { I895, and June 29, I896, } \\
\text { to Sept. 27, I897, con- } \\
\text { stant added }=+\mathrm{I}^{\prime \prime} . \text { So. }\end{array}$} & \multicolumn{4}{|c|}{ Feb. 26, I 895 , to Jan. 4 , I896. } & \multicolumn{4}{|c|}{$\begin{array}{l}\text { Jan. } 27, \text { Igoo, to Nov. I2, } \\
\text { 1900, constant added } \\
=+5^{\prime \prime} .00 .\end{array}$} & \multicolumn{4}{|c|}{$\begin{array}{l}\text { Nov. } 21, \text { I900, to Oct. Io, } \\
\text { 1901, constant added } \\
=+5^{\prime \prime} .00\end{array}$} \\
\hline \multirow[t]{2}{*}{ Z. D.S. } & I & II & III & Z.D.N. & I & II & III & Z.D.N. & I & II & III & Z.D.S. & I & II & III \\
\hline & VII & VI & V & & VII & VI & $\mathrm{V}$ & & VII & VI & $\mathrm{V}$ & & VII & VI & $\mathrm{V}$ \\
\hline$\circ$ & $\prime \prime$ & 11 & 'I & $\circ$ & 'I & $\prime \prime$ & 'I & $\circ$ & "I & 'I & " & $\circ$ & 11 & $\prime \prime$ & 11 \\
\hline 43 & I. 75 & I. 78 & I. 79 & $3 \mathrm{I} 7$ & 0.05 & 0.02 & O. OI & 3 I 7 & 5.05 & 5.02 & 5. Or & 43 & 4.95 & 4.98 & 4. 99 \\
\hline 44 & I. 73 & I. 77 & I. 79 & 316 & 0. 07 & o. 03 & O. oI & 316 & 5.07 & 5.03 & 5. OI & 44 & 4.93 & 4.97 & 4. 99 \\
\hline 45 & I. 72 & I. 76 & I. 79 & $3^{15}$ & o. 08 & 0. 04 & o. oI & 3 I 5 & 5.08 & 5.03 & 5. OI & 45 & 4.92 & 4. 97 & 4. 99 \\
\hline 46 & I. $7 \mathrm{I}$ & I. 76 & I. 79 & 314 & 0.09 & o. 04 & O. OI & 3 I4 & 5.09 & 5.04 & 5.01 & 46 & $4.9 \mathrm{I}$ & 4. 96 & 4. 99 \\
\hline 47 & I. 69 & 1. 75 & I. 79 & $3^{1} 3$ & o. II & o. 05 & O. OI & $3^{1} 3$ & 5. Io & 5.05 & $5 . \mathrm{OI}$ & 47 & 4.90 & 4. 95 & 4. 99 \\
\hline 48 & I. 68 & I. 75 & I. 79 & $3^{12}$ & o. 12 & o. 05 & O. OI & $3 \mathrm{I} 2$ & 5. 12 & 5.05 & 5. OI & 48 & 4. 88 & 4. 95 & 4. 99 \\
\hline 49 & 1.67 & I. 74 & I. 79 & $3 \mathrm{II}$ & o. 13 & 0.06 & O. OI & 3 II & 5. 13 & 5.06 & 5. OI & 49 & 4. 87 & 4. 94 & 4. 99 \\
\hline 50 & I. 65 & I. 73 & I. $7 \mathrm{~S}$ & 310 & o. I5 & 0.07 & 0.02 & 3 Io & 5. I4 & 5. 06 & 5.02 & 50 & 4. 86 & 4. 94 & 4. 98 \\
\hline $5^{I}$ & I. 64 & I. 73 & I. 78 & 309 & o. I6 & 0. 07 & 0.02 & $3^{099}$ & 5. 16 & 5.07 & 5.02 & $5 \mathrm{I}$ & 4. 84 & 4. 93 & 4. 98 \\
\hline 52 & I. 62 & I. 72 & I. 78 & 308 & o. I7 & o. 08 & 0.02 & 308 & 5. 17 & 5. 08 & 5.02 & $5^{2}$ & 4.83 & 4. 92 & 4. 98 \\
\hline 53 & I. $6 \mathrm{r}$ & 1. 72 & I. 78 & 307 & o. I9 & 0.08 & o. 02 & 307 & 5. 19 & 5.08 & 5.02 & 53 & 4. $8 \mathrm{I}$ & 4. 92 & 4.98 \\
\hline 54 & I. 60 & I. $7 \mathrm{I}$ & I. 78 & 306 & o. 20 & 0.09 & 0.02 & 306 & 5. 20 & 5.09 & 5.02 & 54 & 4.80 & 4. $9 \mathrm{I}$ & 4. 98 \\
\hline 55 & I. $5^{8}$ & I. 70 & I. 78 & 305 & o. 22 & o. Io & o. 02 & 305 & 5. $2 \mathrm{I}$ & 5.09 & 5.02 & 55 & 4.79 & 4. $9 \mathrm{I}$ & 4. 98 \\
\hline 56 & I. 57 & I. 70 & I. 77 & 304 & o. 23 & o. IO & 0.03 & 304 & 5.23 & 5. 10 & 5.03 & 56 & 4. 77 & 4. 90 & 4. 97 \\
\hline 57 & I. 55 & I. 69 & I. 77 & 303 & o. 24 & ๑. I I & 0.03 & 303 & 5.24 & 5. II & 5.03 & 57 & $4 \cdot 76$ & 4. 89 & 4. 97 \\
\hline $5^{8}$ & I. 54 & I. 68 & I. 77 & 302 & o. 26 & O. 12 & 0.03 & 302 & 5.25 & 5. II & 5.03 & $5^{8}$ & 4. 75 & 4. 89 & 4. 97 \\
\hline 59 & I. 53 & I. 68 & I. 77 & $3 \mathrm{OI}$ & o. 27 & o. 12 & 0.03 & $3 \mathrm{OI}$ & 5.27 & 5. I 2 & 5.03 & 59 & 4.73 & 4. 88 & 4. 97 \\
\hline 60 & I. $5 \mathrm{I}$ & x. 67 & I. 77 & 300 & o. 29 & O. I3 & 0.03 & 300 & 5.28 & 5. I3 & 5.03 & 60 & 4. 72 & 4. 87 & 4. 97 \\
\hline 61 & 1. $5^{\circ}$ & I. 66 & I. 77 & 299 & 0.30 & o. I4 & 0.03 & 299 & 5.30 & 5. 13 & 5.03 & $6 \mathrm{I}$ & 4.70 & 4. 87 & 4. 97 \\
\hline 62 & I. 48 & I. 66 & I. 76 & 298 & o. 32 & o. I4 & 0.04 & 298 & $5 \cdot 32$ & 5. 14 & 5.03 & 62 & 4.68 & 4. 86 & 4. 97 \\
\hline 63 & I. 46 & I. 65 & I. 76 & 297 & o. 33 & o. I5 & 0.04 & 297 & 5.33 & 5. 15 & 5.04 & $6_{3}$ & 4. 67 & 4. 85 & 4.96 \\
\hline 64 & I. 45 & I. 64 & I. 76 & 296 & o. 35 & o. 16 & 0.04 & 296 & $5 \cdot 35$ & 5. 16 & 5.04 & 64 & 4.65 & 4. 84 & 4. 96 \\
\hline 65 & I. 43 & I. 64 & I. 76 & 295 & o. 37 & o. 16 & o. 04 & 295 & $5 \cdot 36$ & 5. 16 & 5.04 & 65 & 4.64 & 4. 84 & 4. 96 \\
\hline 66 & I. 42 & I. 63 & I. 76 & 294 & 0.38 & o. 17 & O. 04 & 294 & $5 \cdot 3^{8}$ & 5. 17 & 5. 04 & 66 & 4. 62 & 4. 83 & 4. 96 \\
\hline
\end{tabular}


TABLE V.

Precession in Declination from 1894.0 to 1900.0 .

ARGUMEN' $=$ R. A. I894.o.

\begin{tabular}{|c|c|c|c|c|c|c|c|c|c|c|c|c|c|}
\hline $\mathrm{m}$ & $\mathrm{O}^{\mathrm{h}}$ & $\mathrm{I}^{\mathrm{h}}$ & $2^{\mathrm{b}}$ & $3^{\mathrm{h}}$ & $4^{\mathrm{h}}$ & $5^{\mathrm{h}}$ & $6^{\mathrm{h}}$ & $7^{\mathrm{h}}$ & $8^{h}$ & $9^{\mathrm{h}}$ & $10^{\mathrm{h}}$ & $\mathrm{II}^{\mathrm{h}}$ & $\mathrm{m}$ \\
\hline & $\prime \prime$ & "I & " & 'I & '" & $\prime \prime$ & $\prime \prime$ & $\prime \prime$ & " & " & "' & " & \\
\hline $\mathrm{o}$ & $+\mathrm{I} 20.3 \mathrm{I}$ & + II6. I9 & + ro4. I6 & +85.02 & +60.09 & +31.07 & -0.07 & $-3 \mathrm{I} .2 \mathrm{I}$ & -60.22 & -85.13 & -104.23 & -II6. 23 & o \\
\hline $\begin{array}{l}\text { I } \\
2\end{array}$ & $\cdot 3 \mathbf{I}$ & I16.05 & 103.90 & 84.65 & 59.63 & 30.56 & 0.60 & 3I. 72 & 6o. 67 & -50 & .49 & .36 & I \\
\hline 2 & $\cdot 3 \mathrm{I}$ & I I5. 9I & .63 & 84.27 & 59. I7 & 30.05 & I. I2 & 32.22 & 6r. I3 & 85.87 & 104. 75 & .49 & 2 \\
\hline 3 & .30 & .77 & $\cdot 36$ & 83.89 & $5^{8.71}$ & 29.54 & I. 65 & 32. $7 \hat{3}$ & 6 I. $5^{8}$ & 86.24 & 105.01 & .62 & 3 \\
\hline 4 & .29 & .63 & 103.09 & $.5 \mathrm{I}$ & 58.25 & 29.03 & 2. 17 & 33.23 & 62.03 & $.6 \mathrm{I}$ & .26 & .75 & 4 \\
\hline 5 & I 20.27 & $.4 \hat{8}$ & 102.82 & 83.13 & 57.79 & $28.5^{2}$ & 2. $7 \mathrm{O}$ & 33. 74 & .48 & 86.97 & $.5 \mathrm{I}$ & I16. 88 & 5 \\
\hline 6 & .26 & .33 & .54 & 82. 75 & 57.33 & 28.01 & 3.22 & 34. 24 & 62.93 & $87 . \div 3$ & I05. 76 & 117.00 & 6 \\
\hline 7 & .25 & .18 & IO2. 27 & 82. 37 & 56.87 & 27.50 & 3. 75 & 34. 74 & 63.38 & 87.69 & 106. OI & .12 & 7 \\
\hline 8 & .23 & II5. O3 & roI. 99 & 8 r. 99 & $56.4 \mathrm{I}$ & 26. 99 & 4. 27 & 35.25 & 63.82 & 88.05 & .26 & .24 & 8 \\
\hline 9 & $.2 \mathrm{I}$ & II 4.88 & $.7 \mathrm{I}$ & $.6 \mathrm{I}$ & 55.95 & 26. 48 & $4.8 \mathrm{o}$ & 35.75 & 64.27 & $.4 \mathrm{I}$ & $.5 \mathrm{I}$ & $\cdot 36$ & 9 \\
\hline Io & I2O. 19 & .72 & .43 & SI. 22 & .49 & 25.97 & $5 \cdot 3^{2}$ & 55 & $64.7 \mathrm{I}$ & 88.76 & .75 & 117.47 & IO \\
\hline I I & .16 & .56 & IOI. 15 & So. 83 & 55.02 & 25.46 & 5.84 & 36.75 & 65.15 & 89. I I & 106.99 & $.5^{8}$ & II \\
\hline I2 & .14 & .40 & 100.87 & .45 & 54.55 & 24.94 & 6.37 & 37.25 & 65.59 & .47 & 107.23 & .69 & 12 \\
\hline I3 &. $\mathrm{II}$ & .24 & $.5^{8}$ & So. 06 & 54.08 & 24.43 & 6.89 & $37 \cdot 75$ & 66.03 & 89.82 & .47 & .80 & 13 \\
\hline 14 & .07 & I 14.07 & .29 & 79.67 & $53.6 \mathrm{I}$ & 23.91 & $7.4 \mathrm{I}$ & 38.25 & .47 & 90.17 & .70 & I 17.90 & I4 \\
\hline I5 & .04 & II 3.90 & IOO. OO & 79. 28 & 53. I4 & 23.40 & 7.93 & $3^{8.74}$ & 66. 9I & $.5 \mathrm{I}$ & 107.94 & II 8.01 & 15 \\
\hline I6 & 120.00 & .73 & $99.7 \mathrm{I}$ & 78.88 & 52.67 & 22.88 & 8.46 & 39. 24 & 67.35 & 90.86 & 108.17 & . II & 16 \\
\hline 17 & II9. 97 & .56 & .42 & .48 & 52.20 & 22. 37 & 8.98 & 39.74 & 67.78 & 91.20 & .40 & $.2 \mathrm{I}$ & 17 \\
\hline Is & & .39 & 99. I2 & 78.08 & 51. 73 & $2 \mathrm{I} .85$ & 9.50 & 40.23 & $68.2 \mathrm{I}$ & .54 & .62 & $\cdot 30$ & 18 \\
\hline 19 & .88 & $.2 \mathrm{I}$ & 98.82 & 77.68 & 51.26 & 2I. 34 & IO. 03 & 40.73 & 68.64 & 9I. 88 & I08.85 & .40 & 19 \\
\hline 20 & II9. 84 & II 3.03 & .52 & 77.28 & 50.78 & 20.82 & I0. 55 & 4I. 22 & 69.07 & 92. 22 & 109. 07 & I I8. 49 & 20 \\
\hline $2 \mathrm{I}$ & .79 & II 2.85 & 98.22 & 76.88 & 50.30 & 20.30 & II. O7 & 4I. 7I & .50 & .56 & .29 & $.5^{8}$ & $2 \mathrm{I}$ \\
\hline 22 & .74 & .67 & 97.92 & .47 & 49.82 & 19.78 & II. 59 & 42.20 & 69.93 & 92.89 & $.5 \mathrm{I}$ & .67 & 22 \\
\hline 23 & .69 & .48 & $.6 \mathrm{I}$ & 76.07 & & 19.27 & I2. I 2 & 42.69 & 70.35 & 93.22 & .73 & .76 & 23 \\
\hline 24 & .64 & .29 & 97.30 & 75.66 & 48.86 & 18.75 & I 2.64 & 43. 18 & 70.78 & .55 & I09. 94 & .84 & 24 \\
\hline 25 & II9. $5^{8}$ & II2. IO & 96.99 & 75.25 & $48.3^{8}$ & I8. 23 & I3. I6 & 43.67 & 7 I. 20 & 93.88 & I IO. I5 & II8. 92 & 25 \\
\hline 26 & .52 & III. 9I & .68 & 74.84 & 47.90 & $17.7 \mathrm{I}$ & I3. 68 & 44. I 6 & 7 1. 62 & $94.2 \mathrm{I}$ & .36 & I19. oO & 26 \\
\hline 2 & .46 & .72 & .37 & .43 & 47.42 & I7. 19 & I 4.20 & 44.65 & 72.05 & .54 & .57 & .08 & 27 \\
\hline 28 & .40 & .53 & 96.05 & 74. OI & 46.94 & & 14. & & .47 & 94.86 & .78 & .15 & 28 \\
\hline 29 & .34 & .33 & 95.73 & 73.60 & 46.46 & I6. I5 & I5. & & 72.88 & 95.18 & [ 10. 98 & .22 & 29 \\
\hline 30 & II9. 27 & III. I3 & $.4 \mathrm{I}$ & 73. IS & 45.98 & 15.63 & I5. & 46. II & $73 \cdot 30$ & .50 & III. I 8 & II9. 29 & 30 \\
\hline $3 I$ & .20 & I10. 93 & 95.09 & 72.76 & .50 & I5. II & 16. & 46.59 & $73 \cdot 72$ & 95.82 & $\cdot 3^{8}$ & .36 & $3 I$ \\
\hline 32 & .13 & .72 & 94.77 & 2.34 & 45. or & I4. 59 & I6.8I & 47.07 & 74. 13 & 96. I 4 & .58 & .42 & $3^{2}$ \\
\hline 3 & 119.06 & $.5^{\mathrm{I}}$ & .44 & I. 92 & 44.52 & I4. 06 & I 7.33 & 47.55 & .54 & .45 & .77 & .48 & 33 \\
\hline 3 & 8.98 & .30 & 94. II & .50 & 44.03 & 13.54 & I7. 85 & 48.03 & 74.95 & 96.76 & III. 96 & .54 & 34 \\
\hline 35 & .90 & I IO. O9 & 93.78 & 71.08 & 43.54 & I3.02 & I 8. & .51 & $75 \cdot 36$ & 97.07 & II2. I5 & .60 & 35 \\
\hline 3 & .82 & I09. 88 & .45 & 0.65 & 3.05 & $12.5 \mathrm{O}$ & I8. 89 & 48.99 & 75.77 & .38 & $\cdot 34$ & .66 & 36 \\
\hline 37 & .74 & .67 & 93. I 2 & 70.23 & 42.56 & II. 98 & I9. $4 \mathrm{I}$ & 49.47 & 76.18 & 97.69 & .53 & $\cdot 7 \mathrm{I}$ & 37 \\
\hline & .65 & .45 & 92. 79 & 9. So & 42. 07 & II. 45 & 19.92 & 49.95 & $.5^{8}$ & 98.00 & .72 & .76 & $3^{8}$ \\
\hline 3 & .56 & .23 & .46 & 37 & 4I. & & 20.44 & 50.43 & 76.99 & .30 & I I 2.90 & $.8 \mathrm{I}$ & 39 \\
\hline 4 & II 8.47 & IO9. OI & 92. I2 & 68.94 & & $10.4 \mathrm{I}$ & 20.96 & $50.9 \mathrm{I}$ & 77.39 & & I I3. O8 & II9. 86 & 40 \\
\hline 41 & .38 & IOŚ. 79 & 91. 78 & & 0.60 & 89 & 2 I. $4 \mathrm{~S}$ & $5 \mathrm{I}$. & 79 & 98.90 & .26 & $.9 \mathrm{I}$ & $4 \mathrm{I}$ \\
\hline 4 & .28 & .56 & .44 & 68.0 & 40. 10 & $9 \cdot 3^{6}$ & 2 I. 99 & 51.86 & 7 S. 19 & 99.20 & .43 & $\cdot 95$ & 42 \\
\hline 43 & & .34 & 9I. IO & 67.65 & 7. 60 & 8. 84 & & & .59 & .50 & $.6 \mathrm{I}$ & I I9. 99 & 43 \\
\hline 44 & II 8,08 & IOS. II & 90.76 & 67.22 & 39. Io & 8.32 & 23.02 & $5^{2}$. & 78.99 & 99.79 & .78 & 120.03 & 44 \\
\hline 4 & 117.98 & 107. 88 & $.4 \mathrm{I}$ & 66.78 & $3^{9} .6 \mathrm{I}$ & 7.79 & 23.54 & 53.27 & 79.39 & I00. 08 & $\mathrm{II}_{3.95}$ & .06 & 45 \\
\hline & .88 & .64 & 90.06 & 66.34 & $3^{8 .}$ I I & 7.27 & 24.05 & & 79. & .37 & I I4. I I & .09 & 46 \\
\hline 4 & .77 & $.4 \mathrm{I}$ & $89.7 \mathrm{r}$ & 65.90 & $37.6 \mathrm{I}$ & 6.75 & 24.57 & 54. & So. 17 & .66 & .28 & .12 & 47 \\
\hline 4 & .66 & 107.17 & .36 & .46 & & 6.23 & & 54.68 & $\cdot 56$ & 100. 95 & .44 & - 15 & 48 \\
\hline 49 & .55 & 106. 93 & 89.00 & 65.02 & $36.6 \mathrm{I}$ & 5.70 & 25.60 & 55.15 & 8o. 95 & IOI. 23 & .60 & .18 & 49 \\
\hline 5 & I 17.44 & .69 & 88.65 & $64.5^{8}$ & 36. II & & 26. & & S1.34 & $.5 \mathrm{I}$ & .76 & I 20.20 & 50 \\
\hline 5 & .33 & .45 & 88.30 & 64. I4 & $35.6 \mathrm{I}$ & 4. 66 & 26.62 & & SI. 73 ; & IOI. 79 & I I 4.92 & .22 & $5 \mathrm{I}$ \\
\hline $5^{2}$ & $.2 \mathrm{I}$ & 106. 20 & 87.94 & 63.69 & 35. II & 4. 13 & & 56. & 82. I I & IO2. 07 & I 15.07 & .24 & $5^{2}$ \\
\hline 53 & 17.09 & 105.95 & & & 34.60 & $3.6 \mathrm{I}$ & 27. & 57.00 & .49 & $\cdot 35$ & .22 & .26 & 53 \\
\hline 5 & 116.97 & .70 & 87.22 & 62.80 & 34.10 & 3.08 & 28. & .46 & 82.87 & .62 & .37 & .27 & 54 \\
\hline & .85 & .45 & 86.86 & 62. 35 & 33.60 & 2. 56 & 28.66 & 57.92 & $8_{3} .25$ & IO2. S9 & .52 & I 20.28 & 55 \\
\hline 5 & .72 & 105.20 & .50 & $6 \mathrm{I} .90$ & 33.09 & 2.03 & 29. & & 83.63 & IO3. 16 & .67 & .29 & $5^{6}$ \\
\hline 5 & & 104. 94 & 86. I3 & .45 & 32.59 & I. $5 \mathrm{I}$ & 29. & 58.84 & 84.01 & .43 & $.8 \mathrm{r}$ & . 30 & 57 \\
\hline 58 & .46 & .68 & 85.76 & 6I. 00 & 32. 08 & 0.98 & 30. 19 & 59.30 & .39 & $\cdot 70$ & 115.95 & $\cdot 3 \mathrm{I}$ & $5^{8}$ \\
\hline 59 & & .42 & .39 & 60.54 & 3 I. $5^{8}$ & +0.46 & 30.70 & & 84.76 & IO3. 97 & I 16.09 & $.3 \mathrm{I}$ & 59 \\
\hline 60 & & & & +60.09 & +31.07 & -0.07 & $-3 ! \cdot 2 \mathrm{I}$ & -60.22 & -85.13 & -104.23 & -116.23 & $\mid-120.3 \mathrm{I}$ & 60 \\
\hline
\end{tabular}


TABLE V-Continued.

Precession in Declination from 1894.0 to I90o.o-Continued.

ARGUMENT $=$ R. A. IS94.O.

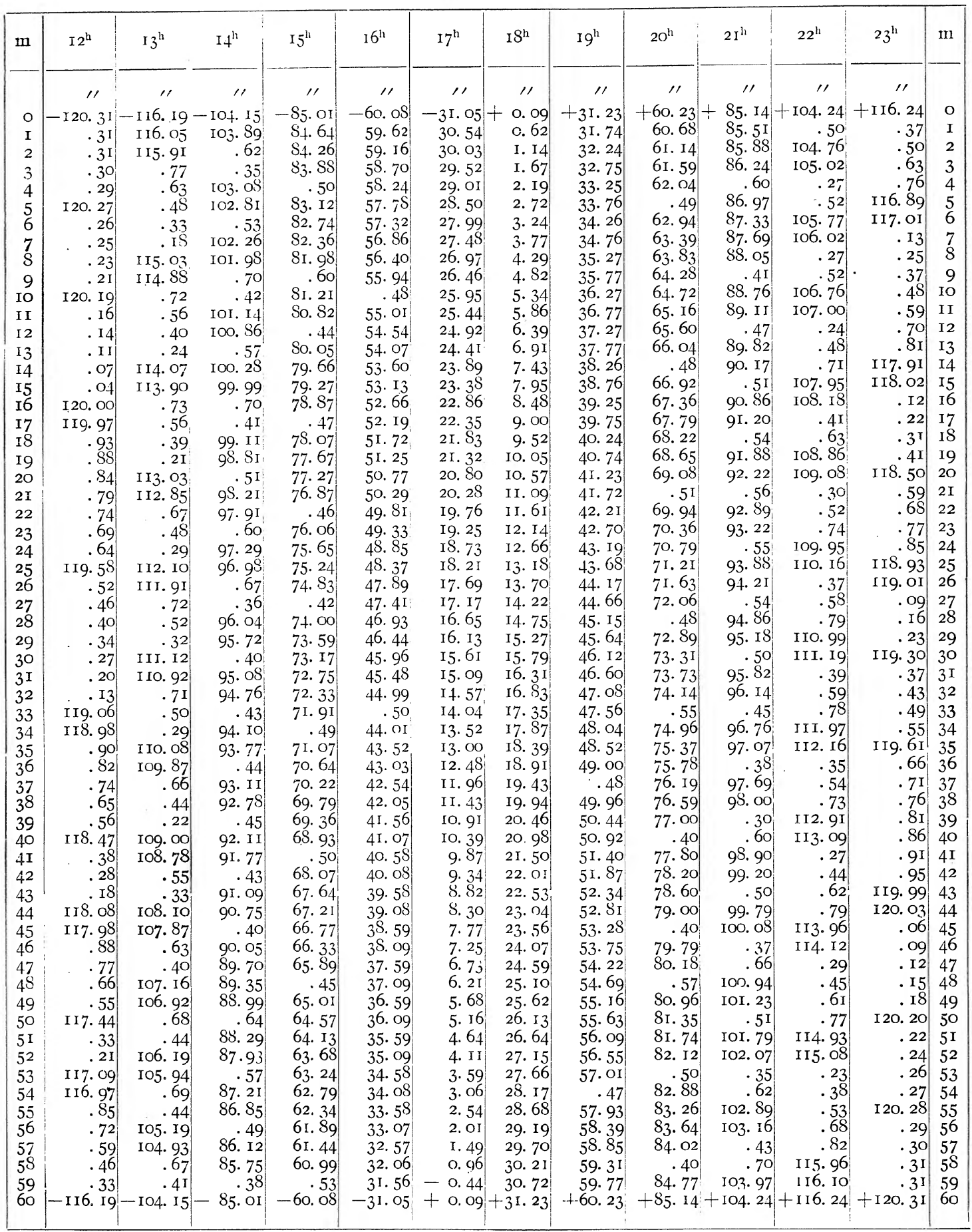


TABLE VI.

Precession in Declination from I895.0 to I900.0.

ARGUMENT $=$ R. A. IS95.o.

\begin{tabular}{|c|c|c|c|c|c|c|c|c|c|c|c|c|c|}
\hline $\mathrm{m}$ & $\mathrm{O}^{\mathrm{h}}$ & $\mathrm{I}^{\mathrm{h}}$ & $2^{\mathrm{h}}$ & $3^{\mathrm{h}}$ & $4^{\mathrm{h}}$ & $5^{\mathrm{h}}$ & $6^{\mathrm{h}}$ & $7^{\mathrm{h}}$ & $8^{h}$ & $9^{1 / 2}$ & $10^{h}$ & $\mathrm{II}^{\mathrm{h}}$ & $\mathrm{m}$ \\
\hline & 11 & 11 & 'I & 'I & 11 & $\prime \prime$ & 11 & '1 & 11 & 11 & 11 & 11 & \\
\hline O & + I00. 26 & +96.83 & +-86.80 & +70.86 & +50.09 & $+25.90-$ & -0.05 & -26.00 & -50.17 & -70.93 & -86.86 & -96.86 & o \\
\hline $\begin{array}{l}\text { I } \\
2\end{array}$ & .26 & $.7 \mathrm{I}$ & $.5 \mathrm{~S}$ & .55 & $49.7 \mathrm{I}$ & .48 & .49 & .42 & .55 & 71.24 & 87.08 & 96.97 & I \\
\hline $\begin{array}{l}2 \\
3\end{array}$ & .26 & .59 & .36 & 70. 24 & 49.33 & 25. 06 & 0.92 & 26.84 & 50.93 & .55 & .29 & 97.08 & 2 \\
\hline 3 & .25 & .47 & 86. 14 & 69.93 & 48.95 & 24.64 & I. 36 & 27.26 & $5 \mathrm{I} .3 \mathrm{O}$ & 71.85 & $.5 \mathrm{I}$ & .19 & 3 \\
\hline $\begin{array}{l}4 \\
5\end{array}$ & .24 & .35 & $85.9 \mathrm{I}$ & .62 & $\cdot 57$ & 24. 22 & I. 80 & 27.68 & 51.68 & 72. I 5 & .72 & .30 & 4 \\
\hline$\frac{5}{6}$ & I00. 23 & .23 & .68 & 69.30 & 48. I9 & 23.80 & 2. 24 & 28. 10 & 52.05 & .45 & 87.93 & 97.40 & 5 \\
\hline $\begin{array}{l}6 \\
7\end{array}$ & $\begin{array}{l}.22 \\
.2 \mathrm{I}\end{array}$ & 96. I I & .45 & 68.98 & $47.8 \mathrm{I}$ & $23 \cdot 37$ & 2.67 & .52 & .42 & 72.75 & 88. I4 & .50 & 6 \\
\hline 8 & $\begin{array}{l}.2 \mathrm{I} \\
.2 \mathrm{O}\end{array}$ & 95.99 & 85.22 & .66 & .42 & 22. 94 & 3. I I & 28. 94 & 52.80 & 73.05 & $\cdot 35$ & .60 & 7 \\
\hline 9 & $\begin{array}{r}.20 \\
.18\end{array}$ & .86 & 84.99 & $\cdot 34$ & 47.03 & $.5 \mathrm{I}$ & .55 & 29.36 & 53. I7 & $\cdot 35$ & .55 & .70 & 8 \\
\hline ro & Ioo. 16 & $\begin{array}{l}.73 \\
.60\end{array}$ & .76 & 68.02 & 46.64 & 22.08 & 3.98 & $29.7 \mathrm{~S}$ & .54 & .65 & $88 \cdot 76$ & $\begin{array}{r}80 \\
80\end{array}$ & 9 \\
\hline I I & .14 & .04 & $\begin{array}{r}.53 \\
.29\end{array}$ & $67.7 \mathrm{O}$ & 46.25 & 21.65 & 4.42 & $\begin{array}{l}30.20 \\
30.62\end{array}$ & $53.9 \mathrm{I}$ & 73.95 & $\begin{array}{l}88.96 \\
80.16\end{array}$ & $\begin{array}{r}.89 \\
97.98\end{array}$ & IO \\
\hline I 2 & . I2 & .34 & $\begin{array}{r}.29 \\
84.05\end{array}$ & $\cdot 3^{8}$ & 45.86 & 2 I. 22 & 4.86 & 30.62 & 54. 28 & 74. 24 & $\begin{array}{r}89.16 \\
.36\end{array}$ & $\begin{array}{l}97.98 \\
98.07\end{array}$ & II \\
\hline I3 & . IO & .20 & $\begin{array}{l}84.05 \\
83.81\end{array}$ & 67.05 & .47 & 20. 79 & 5.29 & 31.03 & 54.65 & $\begin{array}{r}.53 \\
74.82\end{array}$ & $\begin{array}{r}.36 \\
.56\end{array}$ & $\begin{array}{r}98.07 \\
16\end{array}$ & I 2 \\
\hline I4 & .07 & 95.06 & $\begin{array}{r}83.81 \\
.57\end{array}$ & 66. 72 & 45.08 & 20. 37 & $5 \cdot 73$ & .45 & 55. OI & 74. 82 & $\begin{array}{l}.56 \\
.76\end{array}$ & $\begin{array}{l}.16 \\
.25\end{array}$ & I 3 \\
\hline I5 & .04 & 94.92 & $\begin{array}{l}.57 \\
.33\end{array}$ & $\begin{array}{r}\cdot 39 \\
66\end{array}$ & 44.69 & I9. 94 & 6. I7 & 31.86 & .38 & 75. II & $\begin{array}{r}\cdot 76 \\
89 \cdot 95\end{array}$ & $\begin{array}{r}.25 \\
98.34\end{array}$ & $\mathrm{I}_{4}$ \\
\hline I6 & IOO. OI & .78 & $\begin{array}{r}.33 \\
83.09\end{array}$ & $\begin{array}{l}66.06 \\
65.73\end{array}$ & 44.30 & $.5 \mathrm{I}$ & $6.6 \mathrm{I}$ & 32. 28 & 55.74 & $\begin{array}{r}.40 \\
60\end{array}$ & $\begin{array}{l}89.95 \\
\text { 9o. I4 }\end{array}$ & $\begin{array}{r}98.34 \\
.43\end{array}$ & I 5 \\
\hline 17 & 99.98 & .64 & $\begin{array}{l}3.09 \\
82.85\end{array}$ & $\begin{array}{r}65.73 \\
.40\end{array}$ & $43.9 \mathrm{I}$ & I9. 08 & 7.04 & 32.69 & 56. I I & & 90. I4 & .43 & 16 \\
\hline I8 & $\begin{array}{r}.95 \\
. \quad .9\end{array}$ & .50 & .60 & $\begin{array}{r}.40 \\
65.07\end{array}$ & .52 & I8. 65 & .48 & 33. I I & .47 & $\begin{array}{l}75.98 \\
76.27\end{array}$ & $\begin{array}{r}.33 \\
.52\end{array}$ & $.5 \mathrm{I}$ & I7 \\
\hline I9 & .92 & .35 & .35 & $\begin{array}{l}65.07 \\
64.74\end{array}$ & 43.13 & I8. 22 & 7.92 & .52 & 56.83 & $\begin{array}{l}76.27 \\
.55\end{array}$ & $\begin{array}{r}.52 \\
.7 \mathrm{I}\end{array}$ & $\begin{array}{r}59 \\
67\end{array}$ & I8 \\
\hline 20 & .88 & .20 & S2. IO & $\begin{array}{r}64.74 \\
.4 \mathrm{I}\end{array}$ & 42.73 & I 7.79 & S. 35 & 33.93 & 57. I9 & $\begin{array}{r}.55 \\
76.83\end{array}$ & $\begin{array}{r}.7 \mathrm{I} \\
90.89\end{array}$ & .67 & 19 \\
\hline $2 \mathrm{I}$ &.$S_{4}$ & 94.05 & SI. 85 & $\begin{array}{r}.4 \mathrm{I} \\
64.07\end{array}$ & 42.33 & 17.36 & S. 79 & $34 \cdot 34$ & $\cdot 55$ & $\begin{array}{l}76.83 \\
77.11\end{array}$ & $\begin{array}{l}90.89 \\
91.07\end{array}$ & $\begin{array}{r}98.75 \\
83\end{array}$ & 20 \\
\hline 22 & . So & 93.90 & .60 & $\begin{array}{l}64.07 \\
63.73\end{array}$ & 4I. 93 & I6. 93 & 9.22 & 34.75 & $57 \cdot 9 \mathrm{I}$ & $\begin{array}{r}77.11 \\
.39\end{array}$ & $\begin{array}{r}91.07 \\
.25\end{array}$ & $\begin{array}{l}.83 \\
.90\end{array}$ & $2 \mathrm{I}$ \\
\hline 23 & $\cdot 76$ & .74 & .34 & $\begin{array}{r}63.73 \\
.39\end{array}$ & .53 & .50 & 9.66 & 35.16 & 58.27 & $\begin{array}{l}.39 \\
.67\end{array}$ & $\begin{array}{r}.25 \\
.43\end{array}$ & $\begin{array}{r}.90 \\
98.97\end{array}$ & 22 \\
\hline 24 & $.7 \mathrm{I}$ & $.5 \mathrm{~S}$ & 8т. ०8 & $\begin{array}{r}.39 \\
63.05\end{array}$ & 4I. I 3 & I6. 07 & I0. 09 & . $\cdot 57$ & .63 & & $\begin{array}{r}.43 \\
.6 \mathrm{I}\end{array}$ & $\begin{array}{l}98.97 \\
99.04\end{array}$ & 23 \\
\hline 25 & 99.66 & .42 & 80.82 & $\begin{array}{l}63.05 \\
62.7 \mathrm{I}\end{array}$ & 40.73 & I 5.64 & .53 & 35.97 & $5^{8.98}$ & $\begin{array}{l}77.95 \\
78.22\end{array}$ & $\begin{array}{r}.01 \\
.79\end{array}$ & $\begin{array}{r}99.04 \\
\text {. II }\end{array}$ & 24 \\
\hline 26 & $.6 \mathrm{I}$ & .26 & .56 & $\begin{array}{r}62.7 \mathrm{I} \\
.37\end{array}$ & 40.33 & $\mathrm{I} 5.2 \mathrm{I}$ & 10.96 & $36.3^{8}$ & 59.33 & $\begin{array}{r}78.22 \\
.49\end{array}$ & $\begin{array}{r}.79 \\
91.97\end{array}$ & . II & 25 \\
\hline 27 & .56 & .93 .10 & .30 & $\begin{array}{r}\cdot 37 \\
62.03\end{array}$ & 39.93 & I 4.78 & II. 40 & 36.79 & 59.68 & $\begin{array}{r}.49 \\
78.76\end{array}$ & $\begin{array}{l}9^{1} \cdot 97 \\
92.14\end{array}$ & $\begin{array}{r}17 \\
.23\end{array}$ & 26 \\
\hline 28 & .51 & 92.94 & So. 04 & $\begin{array}{l}62.03 \\
61.69\end{array}$ & .53 & I 4.35 & II. 83 & 37.20 & 60.03 & $\begin{array}{l}78.76 \\
79.03\end{array}$ & $\begin{array}{r}92.14 \\
.31\end{array}$ & .23 & 27 \\
\hline 29 & .46 & .78 & 79. 78 & $\begin{array}{r}61.69 \\
.35\end{array}$ & 39. I3 & I3. 92 & I2. 27 & 37.60 & .38 & $\begin{array}{r}79.03 \\
.30\end{array}$ & $\begin{array}{r}.3 \mathrm{I} \\
.48\end{array}$ & .29 & 28 \\
\hline 30 & 99.40 & $.6 \mathrm{I}$ & $.5 \mathrm{I}$ & $\begin{array}{r}\cdot 35 \\
6 \mathrm{r} .00\end{array}$ & 38.73 & .48 & I 2.70 & 38. OI & 60.73 & $\begin{array}{l}.30 \\
.57\end{array}$ & $\begin{array}{l}.48 \\
.65\end{array}$ & $\cdot 35$ & 29 \\
\hline $3 I$ & .34 & .44 & 79. 24 & $\begin{array}{l}6 \mathrm{r} .00 \\
60.65\end{array}$ & 38.32 & I3. 34 & I3. I4 & $.4 \mathrm{I}$ & 61.08 & $\begin{array}{r}.57 \\
79.84\end{array}$ & $\begin{array}{l}.65 \\
.82\end{array}$ & 99. $4 \mathrm{I}_{\mathrm{i}}$ & 30 \\
\hline 32 & .28 & .27 & 78.97 & $\begin{array}{l}60.65 \\
60.30\end{array}$ & $37.9 \mathrm{I}$ & 12.61 & I3. 57 & $3^{8.8 I}$ & .43 & $\begin{array}{l}79.84 \\
80.10\end{array}$ & $\begin{array}{r}.82 \\
92.98\end{array}$ & $\begin{array}{r}.47 \\
52\end{array}$ & $3 I$ \\
\hline 33 & .22 & 92. Io & .70 & $\begin{array}{l}60.30 \\
59.95\end{array}$ & $.5 \mathrm{I}$ & I2. I7 & $\mathrm{I} 4.0 \mathrm{I}$ & $39.2 \mathrm{I}$ & $6 \mathrm{r} .77$ & $\begin{array}{r}\text { 80. I0 } \\
.36\end{array}$ & $\begin{array}{l}92.98 \\
93.14\end{array}$ & $\cdot 5^{2}$ & 32 \\
\hline 34 & .16 & 91.92 & .43 & $\begin{array}{r}59.95 \\
.60\end{array}$ & 37.10 & II. 74 & .44 & $39.6 \mathrm{I}$ & 62. II & $\begin{array}{r}.36 \\
.62\end{array}$ & & .57 & 33 \\
\hline 35 & .09 & .74 & 78.16 & $\begin{array}{r}.60 \\
59.25\end{array}$ & 36. 69 & II. 30 & I4. 87 & $40.0 \mathrm{I}$ & .45 & $\begin{array}{r}.62 \\
\text { So. } 88\end{array}$ & $\begin{array}{r}\cdot 30 \\
.46\end{array}$ & .62 & 34 \\
\hline 36 & 99.02 & .56 & 77.89 & $\begin{array}{l}59.25 \\
58.90\end{array}$ & 36.29 & IO. 87 & 15.30 & $.4 \mathrm{I}$ & 62.79 & $\begin{array}{l}80.88 \\
\text { SI. 14. }\end{array}$ & $\begin{array}{l}.46 \\
.62\end{array}$ & 99.67 & 35 \\
\hline 37 & 98.95 & .38 & $.6 \mathrm{I}$ & $\begin{array}{r}58.90 \\
.55\end{array}$ & 35.88 & .43 & I 5.74 & $40.8 \mathrm{I}$ & 63.13 & $\begin{array}{r}\text { SI. I4 } \\
.40\end{array}$ & $\begin{array}{l}.62 \\
.78\end{array}$ & .72 & $3^{6}$ \\
\hline 38 & .88 & .20 & .33 & $\begin{array}{r}.55 \\
5 \text { S. I9 }\end{array}$ & .47 & IO. 00 & 16. I7 & $4 \mathrm{I} .2 \mathrm{I}$ & .47 & $\begin{array}{l}.40 \\
.66\end{array}$ & $\begin{array}{r}.78 \\
93.93\end{array}$ & $\cdot 76$ & 37 \\
\hline 39 & $.8 \mathrm{I}$ & 91.02 & 77.05 & $\begin{array}{l}58.19 \\
57.83\end{array}$ & 35.06 & 9.56 & I6. 60 & 4I. 6I & $63.8 \mathrm{I}$ & $\begin{array}{r}.66 \\
\text { Si. } 91\end{array}$ & $\begin{array}{l}93.93 \\
94.08\end{array}$ & $\begin{array}{l}. \mathrm{SO}_{1} \\
. \mathrm{S}_{4}\end{array}$ & $3^{8}$ \\
\hline 40 & .73 & 90.84 & 76.77 & $\begin{array}{r}57.83 \\
.47\end{array}$ & 34.65 & 9.13 & 17.03 & 42. OI & 64.15 & $\begin{array}{l}\text { SI. 9I } \\
\text { 82. I } 6\end{array}$ & $\begin{array}{r}94.00 \\
.23\end{array}$ & & 39 \\
\hline 41 & .65 & .65 & .49 & $\begin{array}{r}.47 \\
57 . \\
\text { I I }\end{array}$ & 34. 24 & 8.69 & .46 & $.4 \mathrm{I}$ & .49 & $\begin{array}{r}82.16 \\
.4 \mathrm{I}\end{array}$ & $.3 \mathrm{~S}$ & $\begin{array}{r}99.88 \\
.92\end{array}$ & 40 \\
\hline 42 & .57 & .46 & 76.21 & $\begin{array}{l}57.1 \mathrm{I} \\
56.75\end{array}$ & 33.83 & 8. 26 & I 7. 89 & 42. $8 \mathrm{I}$ & 64.82 & $\begin{array}{l}.4 \mathrm{I} \\
.66\end{array}$ & .53 & $\begin{array}{l}.92 \\
.95\end{array}$ & 4I \\
\hline 43 & .49 & .27 & 75.92 & $\begin{array}{r}56.75 \\
.39\end{array}$ & .42 & 7.83 & I 8. $3 \mathrm{I}$ & 43.20 & 65.15 & $\begin{array}{r}.66 \\
82.91\end{array}$ & .67 & $\begin{array}{r}.95 \\
99.98\end{array}$ & 42 \\
\hline 44 & $.4 \mathrm{I}$ & 90.08 & .63 & $\begin{array}{r}.39 \\
56.03\end{array}$ & $33 . \mathrm{OI}$ & 7.40 & I8. 74 & .59 & $6=.48$ & $\begin{array}{l}82.91 \\
83.15\end{array}$ & $.8 \mathrm{I}$ & $\begin{array}{r}99.9^{8} \\
\text { IOO. OI }\end{array}$ & 43 \\
\hline 45 & 98.32 & 89.89 & .34 & $\begin{array}{l}56.03 \\
55.67\end{array}$ & 32.60 & 6.96 & I9. I 7 & 43.99 & $65.8 \mathrm{I}$ & $\begin{array}{r}3.15 \\
.39\end{array}$ & 94.95 & $\begin{array}{r}\text { I00. OI } \\
.04\end{array}$ & 44 \\
\hline 46 & .23 & .70 & 75.05 & $\begin{array}{l}55.67 \\
55.3 \mathrm{I}\end{array}$ & 32. 19 & .52 & 19.60 & $44 \cdot 38$ & 66. 14 & $\begin{array}{r}.39 \\
.63\end{array}$ & 95.09 & $\begin{array}{l}.04 \\
.07\end{array}$ & 45 \\
\hline 47 & . I4 & $.5 \mathrm{I}$ & 74.76 & $\begin{array}{l}55 \cdot 3 \mathrm{I} \\
54.94\end{array}$ & 3I. 78 & 6. o8 & 20. 03 & $44 \cdot 77$ & $\begin{array}{r}.47 \\
66.80\end{array}$ & 83.83 & $\begin{array}{r}.23 \\
\end{array}$ & $\begin{array}{l}.07 \\
.10\end{array}$ & 46 \\
\hline 48 & 98.05 & $.3 \mathrm{I}$ & .47 & $\begin{array}{r}54.94 \\
.57\end{array}$ & $3 \mathrm{I} \cdot 36$ & 5.64 & .46 & 45.16 & 66.80 & $\begin{array}{l}83.87 \\
84 . \text { I I }\end{array}$ & .37 & $\begin{array}{l}. \mathrm{IO} \\
. \mathrm{I} 3\end{array}$ & 47 \\
\hline 49 & 97.96 & 89. II & 74. IS & $\begin{array}{r}\cdot 57 \\
54.20\end{array}$ & 30.94 & 5.20 & 20. S9 & .55 & 67.13 & $\begin{array}{r}84.11 \\
.35\end{array}$ & $.5 \mathrm{I}$ & $\begin{array}{l}.13 \\
.15\end{array}$ & 48 \\
\hline 50 & .87 & $88.9 \mathrm{I}$ & 73.88 & $\begin{array}{l}54.2 \mathrm{O} \\
53.83\end{array}$ & $\cdot 5^{2}$ & 4. 76 & $2 \mathrm{I} .32$ & 45.94 & .45 & $\begin{array}{r}.35 \\
.59\end{array}$ & .64 & $\begin{array}{r}.15 \\
\text { I00. } 17\end{array}$ & 49 \\
\hline $5 \mathrm{I}$ & .77 & $.7 \mathrm{I}$ & .58 & $\begin{array}{r}53.8_{3} \\
.46\end{array}$ & 30. Io & 4. 32 & 2 I. 75 & 46.33 & 67.77 & $\begin{array}{r}.59 \\
84.82\end{array}$ & .77 & $\begin{array}{r}\text { I00. } 17 \\
.19\end{array}$ & $5^{\circ}$ \\
\hline $5^{2}$ & .67 & .50 & 73.28 & $\begin{array}{r}.46 \\
53.09\end{array}$ & 29.68 & 3.88 & 22. IS & 46. 72 & 68. 09 & $\begin{array}{l}84.02 \\
85.05\end{array}$ & 95.90 & $\begin{array}{l}.19 \\
.2 \mathrm{I}\end{array}$ & $5^{I}$ \\
\hline 53 & .57 & .29 & 72.98 & $\begin{array}{l}53.09 \\
52.72\end{array}$ & 29. 26 & .45 & 22.60 & 47. I I & $68^{-41}$ & $\begin{array}{r}05.05 \\
.28\end{array}$ & 96.03 & $\begin{array}{l}.2 \mathrm{I} \\
.22\end{array}$ & $5^{2}$ \\
\hline 54 & .47 & 88. 08 & .68 & $\begin{array}{l}52.72 \\
52.35\end{array}$ & 28.84 & 3. OI & 23.03 & .49 & 68.73 & $\begin{array}{l}.2 \mathrm{O} \\
.5 \mathrm{I}\end{array}$ & $\begin{array}{r}\mathrm{I} \\
\end{array}$ & $\begin{array}{l}.22 \\
.23\end{array}$ & 53 \\
\hline 55 & 97.37 & 87.87 & .38 & $\begin{array}{l}\text { 52. } 35 \\
5 \text { I. } 98\end{array}$ & .42 & 2. 57 & .46 & 47.88 & 69.05 & $\begin{array}{r}.51 \\
.74\end{array}$ & $\begin{array}{r}.27 \\
.27\end{array}$ & $\begin{array}{r}.23 \\
\text { IOO. } 24\end{array}$ & 54 \\
\hline 56 & .27 & .66 & 72. o8 & $\begin{array}{r}5 \text { I. } 98 \\
.6 \mathrm{I}\end{array}$ & $2 \mathrm{~S} . \mathrm{OO}$ & 2. I4 & 23. 88 & 48.26 & $\cdot 37$ & 85.97 & .39 & $\begin{array}{r}\text { IOO. } 24 \\
.25\end{array}$ & 55 \\
\hline 57 & . I6 & .45 & 71.78 & $\begin{array}{r}.6 \mathrm{I} \\
5 \mathrm{I} .23\end{array}$ & $27.5 \mathrm{~S}$ & I. 70 & $24.3 \mathrm{I}$ & 48.64 & 69.69 & $\begin{array}{l}35.97 \\
86.20\end{array}$ & $.5 \mathrm{I}$ & .25 & 56 \\
\hline $5^{8}$ & & .24 & .48 & $\begin{array}{l}5 \mathrm{I} .23 \\
5 \mathrm{O} .85\end{array}$ & 27. I6 & I. 26 & 24. 73 & 49.03 & 70. 00 & $\begin{array}{r}00.20 \\
.42\end{array}$ & .63 & .2 & 57 \\
\hline 59 & 96. 94 & 87.02 & 7I. I7 & $\begin{array}{r}50.85 \\
.47\end{array}$ & 26. 74 & O. $\mathrm{S2}$ & 25. I5 & $.4 \mathrm{I}$ & $\cdot 3 \mathrm{I}$ & $\begin{array}{r}.42 \\
.64\end{array}$ & .75 & .26 & $5^{8}$ \\
\hline 60 & +96.83 & +86.80 & +70.86 & $\begin{array}{r}.47 \\
+50.09\end{array}$ & $\begin{array}{r}26.32 \\
\end{array}$ & +0.39 & 25.58 & $\begin{array}{r}49.79 \\
-50.17\end{array}$ & .02 & -86.86 & -96.86 & $\begin{array}{r}.26 \\
-100.26\end{array}$ & 59 \\
\hline & & & & +50.09 & +25.90 & -0.05 & $26.00_{1}$ & -50.17 & .95 & & & & \\
\hline
\end{tabular}


TABLE VI-Continued.

Precession in Declination from 1895.0 to $1900.0-$ Continued.

ARGUMENT $=$ R. A. I895.o.

\begin{tabular}{|c|c|c|c|c|c|c|c|c|c|c|c|c|c|}
\hline $\mathrm{m}$ & $12^{\mathrm{h}}$ & $\mathrm{I} 3^{\mathrm{h}}$ & $I 4^{\mathrm{h}}$ & $15^{\mathrm{h}}$ & $16^{\mathrm{h}}$ & $17^{\mathrm{h}}$ & I $8^{\mathrm{h}}$ & $19^{\mathrm{h}}$ & $20^{h}$ & $2 \mathrm{I}^{\mathrm{h}}$ & $22^{\mathrm{h}}$ & $23^{\mathrm{h}}$ & $\mathrm{m}$ \\
\hline & 11 & 11 & "I & II & 11 & $\prime \prime$ & $\prime \prime$ & II & 11 & 11 & "I & II & \\
\hline o & - I00. 26 & $-96.8_{3}$ & -86.80 & -70.85 & -50.08 & -25.89 & +0.06 & $+26.0 \mathrm{I}$ & +50.18 & +70.94 & +86.86 & +96.86 & o \\
\hline I & .26 & $\cdot 7 \mathrm{I}$ & .58 & .54 & 49. 70 & .47 & .50 & $\begin{array}{r}.43 \\
2685\end{array}$ & $\begin{array}{r}.56 \\
50.94\end{array}$ & 71. 25 & 87.08 & $\begin{array}{l}96.97 \\
97.08\end{array}$ & $\begin{array}{l}\mathrm{I} \\
2\end{array}$ \\
\hline 2 & .26 & $\begin{array}{r}.59 \\
.47\end{array}$ & $\begin{array}{r}\cdot 36 \\
86.14\end{array}$ & 70. 23 & $\begin{array}{l}49 \cdot 32 \\
48.94\end{array}$ & $\begin{array}{l}25.05 \\
24.63\end{array}$ & $\begin{array}{l}\text { O. } 93 \\
\text { I. } 37\end{array}$ & $\begin{array}{l}26.85 \\
27.27\end{array}$ & $\begin{array}{l}50.94 \\
5 \text { I. } 3 \mathrm{I}\end{array}$ & $\begin{array}{r}.56 \\
71.86\end{array}$ & $\begin{array}{l}.29 \\
.5 \mathrm{I}\end{array}$ & $\begin{array}{r}97.00 \\
.19\end{array}$ & $\begin{array}{l}2 \\
3\end{array}$ \\
\hline $\begin{array}{l}3 \\
4\end{array}$ & $\begin{array}{r}25 \\
24\end{array}$ & $\begin{array}{l}.47 \\
.35\end{array}$ & $\begin{array}{l}86.14 \\
85.91\end{array}$ & $\begin{array}{r}69.92 \\
.6 \mathrm{I}\end{array}$ & $\begin{array}{r}48.94 \\
.56\end{array}$ & $\begin{array}{l}24.03 \\
24.21\end{array}$ & $\begin{array}{l}\text { I. } 37 \\
\text { I. } 8 \mathrm{I}\end{array}$ & $\begin{array}{l}27.27 \\
27.69\end{array}$ & 5I. 69 & 72. 16 & .72 & .30 & 4 \\
\hline $\begin{array}{l}4 \\
5\end{array}$ & $\begin{array}{r}.24 \\
\text { I00. } 23\end{array}$ & $\begin{array}{r}.35 \\
.23\end{array}$ & $\begin{array}{r}05.91 \\
.68\end{array}$ & 69.29 & 48. I8 & 23.79 & 2. 25 & 28. II & 52.06 & .46 & 87.93 & 97.40 & 5 \\
\hline 6 & $\begin{array}{r}100.23 \\
.22\end{array}$ & 96. II & .45 & 68.97 & 47.80 & $23 \cdot 36$ & 2. 68 & .53 & .43 & 72. 76 & 88. I4 & .50 & 6 \\
\hline 7 & $.2 \mathrm{I}$ & 95.99 & 85.22 & .65 & $.4 \mathrm{I}$ & 22.93 & 3. I2 & 28. 95 & $52.8 \mathrm{I}$ & 73.06 & $\cdot 35$ & .60 & 7 \\
\hline $\begin{array}{l}8 \\
9\end{array}$ & $\begin{array}{r}20 \\
18\end{array}$ & .86 & 84.99 & .33 & 47.02 & .50 & $\cdot 56$ & 29. 37 & 53. I8 & .36 & $\cdot 55$ & . 70 & 8 \\
\hline $\begin{array}{r}9 \\
\text { I0 }\end{array}$ & $\begin{array}{r}.18 \\
\text { I00. } 16\end{array}$ & $\begin{array}{l}.73 \\
.60\end{array}$ & $\begin{array}{l}.76 \\
.53\end{array}$ & $\begin{array}{l}68.01 \\
67.69\end{array}$ & 46.63 & $\begin{array}{l}22.07 \\
21.64\end{array}$ & $\begin{array}{l}3.99 \\
4.43\end{array}$ & $\begin{array}{l}29.79 \\
30.2 \mathrm{I}\end{array}$ & $\begin{array}{r}.55 \\
53.92\end{array}$ & $\begin{array}{r}.66 \\
73.96\end{array}$ & $\begin{array}{r}.76 \\
88.96\end{array}$ & $\begin{array}{l}.80 \\
.89\end{array}$ & $\begin{array}{r}9 \\
\text { I0 }\end{array}$ \\
\hline I I & $\begin{array}{r}\text { I00. } 16 \\
.14\end{array}$ & $\begin{array}{l}.00 \\
.47\end{array}$ & $\begin{array}{l}.53 \\
.29\end{array}$ & $\begin{array}{r}67.69 \\
.37\end{array}$ & $\begin{array}{l}46.24 \\
45.85\end{array}$ & $\begin{array}{l}21.04 \\
21.2 \mathrm{I}\end{array}$ & $\begin{array}{l}4.43 \\
4.87\end{array}$ & $\begin{array}{l}50.61 \\
30.63\end{array}$ & $\begin{array}{l}53.92 \\
54.29\end{array}$ & $\begin{array}{l}73.90 \\
74.25\end{array}$ & 89. I6 & $\begin{array}{r}.89 \\
97.98\end{array}$ & $\begin{array}{l}10 \\
\text { I I }\end{array}$ \\
\hline I2 & .12 & .34 & 84.05 & 67.04 & .46 & 20.78 & 5.30 & 3 I. 04 & 54.66 & $\begin{array}{r}.54 \\
\end{array}$ & .36 & 98. 07 & 12 \\
\hline I3 & . IO & .20 & $83.8 \mathrm{I}$ & $66.7 \mathrm{I}$ & 45.07 & 20. 35 & 5.74 & .46 & 55.02 & 74.83 & .56 & .16 & I3 \\
\hline 14 & .07 & 95.05 & .57 & $.3^{8}$ & 44.68 & I9. 92 & 6. 18 & 3 I. 87 & .39 & 75. I2 & $\cdot 76$ & .25 & I4 \\
\hline 15 & .04 & 94.92 & .33 & 66.05 & 44. 29 & .49 & 6.62 & 32.29 & 55.75 & .41 & 89.95 & .34 & 15 \\
\hline I6 & IOO. OI & .78 & 83.09 & 65.72 & 43.90 & 19. 06 & 7.05 & 32.70 & 56. 12 & .70 & 90. I4 & .43 & 16 \\
\hline I 7 & 99.98 & .64 & 82.85 & .39 & $.5 \mathrm{I}$ & I8. 63 & .49 & 33. 12 & .48 & 75.99 & .33 & $98.5 \mathrm{I}$ & I 7 \\
\hline I8 & .95 & .50 & .60 & 65.06 & 43. I2 & I8. 20 & 7.93 & .53 & 56.84 & 76. 28 & .52 & .59 & 18 \\
\hline 19 & $\cdot 92$ & $\cdot 35$ & .35 & 64.73 & 42.72 & I 7. 77 & 8.36 & 33.94 & 57.20 & .56 & $.7 \mathrm{I}$ & .67 & I9 \\
\hline 20 & .88 & .20 & 82. IO & .40 & 42.32 & I7. 34 & 8.80 & 34.35 & $.5^{6}$ & 76.84 & 90.89 & .75 & 20 \\
\hline $2 I$ & .84 & 94.05 & 81. 85 & 64.06 & 41.92 & I6. 9I & 9.23 & 34.76 & $57 \cdot 92$ & 77.12 & 91.07 & .83 & $2 \mathrm{I}$ \\
\hline 22 & .80 & 93.90 & .60 & 63.72 & .52 & .48 & 9.67 & 35.17 & 59.27 & .40 & .25 & .90 & 22 \\
\hline 23 & . 76 & .74 & $\cdot 34$ & $\cdot 3^{8}$ & 4I. I2 & I6. 05 & IO. IO & $\cdot 5^{8}$ & .63 & .68 & .43 & 98.97 & 23 \\
\hline 24 & $\cdot 7 \mathrm{I}$ & .58 & 81. o8 & 63.04 & 40.72 & 15.62 & .54 & 35.99 & 58.98 & 77.96 & $.6 \mathrm{I}$ & 99.04 & 24 \\
\hline 25 & 99.66 & .42 & 80.82 & 62.70 & 40.32 & I5. I9 & I0. 97 & 36.40 & 59.33 & 78.23 & .79 & . I I & 25 \\
\hline 26 & $.6 \mathrm{I}$ & .26 & $\cdot 5^{6}$ & .36 & 39.92 & I4. 76 & II. $4 \mathrm{I}$ & 36.80 & 59.68 & .50 & 91. 97 & . I7 & 26 \\
\hline 27 & $\cdot 56$ & 93. Io & .30 & 62.02 & .52 & I4. 33 & II. 84 & $37.2 \mathrm{I}$ & 60. 04 & 78.77 & 92. 14 & .23 & 27 \\
\hline 28 & $.5 \mathrm{I}$ & 92.94 & 80. 04 & 61.68 & 39. I2 & I3. 90 & 12. 28 & 37.62 & .39 & 79.04 & $.3 \mathrm{I}$ & .29 & 28 \\
\hline 29 & .46 & . 78 & 79. 78 & $6 r .34$ & 38.72 & .46 & I2. $7 \mathrm{I}$ & 38.02 & 60.73 & $\cdot 3 \mathrm{I}$ & .48 & .35 & 29 \\
\hline 30 & 99.40 & $.6 \mathrm{I}$ & $.5 \mathrm{I}$ & 60.99 & $38.3 \mathrm{I}$ & 13.02 & I3. I5 & .43 & 61.08 & .58 & .65 & $99.4 \mathrm{I}$ & 30 \\
\hline $3 I$ & .34 & .44 & 79. 24 & .64 & 37.90 & I2. 59 & I3. $5^{8}$ & 38.83 & .43 & 79.84 & .82 & .47 & $3 I$ \\
\hline 32 & .28 & .27 & 78.97 & 60.29 & .50 & 12. 15 & I4. 02 & 39.23 & 6 I. 77 & $80 . \mathrm{II}$ & 92.98 & .52 & 32 \\
\hline 33 & .22 & 92. Io & .70 & 59.94 & 37.09 & II. 72 & .45 & 39.63 & 62. II & $\cdot 37$ & 93. I4 & .57 & 33 \\
\hline 34 & .16 & 91.92 & .43 & .59 & 36.68 & II. 28 & I4. 88 & 40. 03 & .45 & .63 & .30 & .62 & 34 \\
\hline 35 & .09 & .74 & 78.16 & 59.24 & 36.28 & IO. 85 & $\mathrm{I} 5 \cdot 3^{\mathrm{I}}$ & .43 & 62.79 & 80.89 & .46 & .67 & 35 \\
\hline 36 & 99.02 & .56 & 77.89 & 58.89 & 35.87 & 10. $4 \mathrm{I}$ & I5. 75 & 40.83 & 63.13 & 8I. I4 & .62 & .72 & 36 \\
\hline 37 & 98. 95 & .38 & $.6 \mathrm{I}$ & .54 & .46 & 9.98 & I6. 18 & 4I. 23 & .47 & .40 & .78 & 99.76 & 37. \\
\hline $3^{8}$ & .88 & .20 & .33 & 58. I8 & 35.05 & .54 & I6. $6 \mathrm{I}$ & 41.63 & $63.8 \mathrm{I}$ & .66 & $93 \cdot 93$ & .80 & $3^{8}$ \\
\hline 39 & $.8 \mathrm{I}$ & 91.02 & 77.05 & 57.82 & 34.64 & 9. I I & 17.04 & 42.03 & 64.15 & 8I. 9I & 94.08 & .84 & 39 \\
\hline 40 & 98.73 & 90.84 & 76. 77 & .46 & 34. 23 & 8.67 & .47 & .43 & .49 & 82. 16 & .23 & .88 & 40 \\
\hline $4 \mathrm{I}$ & .65 & .65 & .49 & 57. Io & 33.82 & 8.23 & 17.90 & 42.83 & 64.82 & . 4I & $\cdot 38$ & .92 & $4 \mathrm{I}$ \\
\hline $4^{2}$ & $\cdot 57$ & .46 & $76.2 \mathrm{I}$ & 56. 74 & $.4 \mathrm{I}$ & 7.80 & I8. 32 & 43.22 & 65.16 & .66 & .53 & .95 & 42 \\
\hline 43 & .49 & .27 & $75.9^{2}$ & $.3^{8}$ & 33.00 & $7 \cdot 36$ & I8. 75 & $43.6 \mathrm{I}$ & .49 & $82.9 \mathrm{I}$ & .67 & 99.98 & 43 \\
\hline 44 & $.4 \mathrm{I}$ & 90.08 & .63 & 56. O2 & 32.59 & 6. 93 & 19. I8 & 44. OI & 65.82 & 83.15 & $.8 \mathrm{I}$ & IOO. OI & 44 \\
\hline 45 & $9^{8} \cdot 3^{2}$ & 89.89 & .34 & 55.66 & 32. 18 & .49 & I9. $6 \mathrm{I}$ & .40 & 66. I5 & .39 & 94.95 & .04 & 45 \\
\hline 46 & .23 & .70 & 75.05 & $55 \cdot 30$ & 31.77 & 6.05 & 20. 04 & 44. 79 & .48 & .63 & 95.09 & .07 & 46 \\
\hline 47 & .14 & $.5 \mathrm{I}$ & $74 \cdot 76$ & 54. 93 & 31.35 & 5.62 & .47 & 45. 18 & 66. SI & 83.87 & .23 & . IO & 47 \\
\hline 48 & 98.05 & $.3 \mathrm{I}$ & .47 & .56 & 30.93 & 5. 18 & 20.90 & .57 & 67.13 & 84. II & .37 & .13 & 48 \\
\hline 49 & 97.96 & 89. II & 74. 18 & 54. I9 & $.5 \mathrm{I}$ & $4 \cdot 75$ & 2I. 33 & 45.95 & .46 & $\cdot 35$ & $.5 \mathrm{I}$ & .15 & 49 \\
\hline 50 & .87 & 88. $9 \mathrm{I}$ & 73.88 & 53.82 & 30.09 & $4 \cdot 3 \mathrm{I}$ & 2 r. 76 & 46.34 & 67.78 & .59 & .64 & I00. 17 & $5^{\circ}$ \\
\hline $5 \mathrm{I}$ & .77 & $.7 \mathrm{I}$ & $.5^{8}$ & .45 & 29.67 & 3.87 & 22. 19 & 46. 73 & 68. 10 & 84.82 & .77 & . I9 & $5 \mathrm{I}$ \\
\hline 52 & .67 & .50 & 73.28 & 53.08 & 29. 25 & .44 & $22.6 \mathrm{I}$ & 47. I2 & .42 & 85.05 & 95.90 & $.2 \mathrm{I}$ & 52 \\
\hline 53 & .57 & .29 & 72. 98 & $52.7 \mathrm{I}$ & 28.83 & 3.00 & 23.04 & .50 & 68.74 & .28 & 96. O3 & .22 & 53 \\
\hline 54 & .47 & 88.08 & .68 & 52.34 & $28.4 \mathrm{I}$ & 2. 56 & .47 & 47.89 & 69.06 & $.5 \mathrm{I}$ & .15 & .23 & 54 \\
\hline 55 & $97 \cdot 37$ & 87.87 & $\cdot 38$ & 51. 97 & 27.99 & 2. I3 & 23.89 & 48.27 & .38 & .74 & .27 & .24 & 55 \\
\hline $5^{6}$ & .27 & .66 & 72. 08 & .60 & .57 & I. 69 & $24 \cdot 32$ & 48.65 & 69.70 & 85.97 & .39 & IOO. 25 & 56 \\
\hline 57 & .16 & .45 & 71.78 & 5I. 22 & 27.15 & I. 25 & 24.74 & 49. 04 & 70. OI & 86. 20 & $.5 \mathrm{I}$ & .26 & 57 \\
\hline $5^{8}$ & 97.05 & .24 & .47 & 50. 84 & 26. 73 & $0.8 \mathrm{I}$ & 25. 16 & .42 & .32 & .42 & .63 & .26 & $5^{8}$ \\
\hline 59 & 96. 94 & 87.02 & 71.16 & .46 & 26. 3I & -0.38 & 25.59 & 49. 80 & .63 & & .75 & $5 \quad .26$ & 59 \\
\hline 60 & -96.83 & -86.80 & -70.85 & -50.08 & -25.89 & +0.06 & $+26.0 \mathrm{I}$ & +50.18 & +70.94 & -86.86 & -96.86 & $6+100.26$ & 60 \\
\hline
\end{tabular}

